\title{
Combinatorics of the free Baxter algebra
}

\author{
Marcelo Aguiar* \\ Department of Mathematics \\ Texas A\&M University, College Station, TX, USA \\ maguiar@math. tamu.edu \\ Walter Moreira* \\ Department of Mathematics \\ Texas A\&M University, College Station, TX, USA \\ wmoreira@math.tamu.edu
}

Submitted: Oct 7, 2005; Accepted: Feb 9, 2006; Published: Feb 22, 2006

Mathematics Subject Classification: 05A15, 08B20, 16W99

\begin{abstract}
We study the free (associative, non-commutative) Baxter algebra on one generator. The first explicit description of this object is due to Ebrahimi-Fard and Guo. We provide an alternative description in terms of a certain class of trees, which form a linear basis for this algebra. We use this to treat other related cases, particularly that in which the Baxter map is required to be quasi-idempotent, in a unified manner. Each case corresponds to a different class of trees.

Our main focus is on the underlying combinatorics. In several cases, we provide bijections between our various classes of trees and more familiar combinatorial objects including certain Schröder paths and Motzkin paths. We calculate the dimensions of the homogeneous components of these algebras (with respect to a bidegree related to the number of nodes and the number of angles in the trees) and the corresponding generating series. An important feature is that the combinatorics is captured by the idempotent case; the others are obtained from this case by various binomial transforms. We also relate free Baxter algebras to Loday's dendriform trialgebras and dialgebras. We show that the free dendriform trialgebra (respectively, dialgebra) on one generator embeds in the free Baxter algebra with a quasi-idempotent map (respectively, with a quasi-idempotent map and an idempotent generator). This refines results of Ebrahimi-Fard and Guo.
\end{abstract}

*Both authors supported in part by NSF grant DMS-0302423. We thank Kurusch Ebrahimi-Fard for an explanation of the paper [6], which led us to the results of this paper. 


\section{Introduction}

A Baxter algebra (also called Rota-Baxter algebra in some of the recent literature) is a pair $(A, \beta)$ consisting of an associative algebra $A$ and a linear map $\beta: A \rightarrow A$ satisfying

$$
\beta(a) \beta(b)=\beta(\beta(a) b+a \beta(b)+\lambda a b),
$$

where $\lambda$ is a fixed scalar. Interest in these objects originated in work of Baxter [2]. Constructing the free Baxter algebra in explicit terms amounts to describing all consequences of the above identity.

Rota gave the first description of the free commutative Baxter algebra [12], by providing an embedding into an explicit Baxter algebra. Cartier then obtained an intrinsic description [4]. For other references to early work, see [13, 14]. More recently, Guo and Keigher described the adjoint functor to the forgetful functor from the category of commutative Baxter algebras to the category of commutative algebras [8].

It is natural to consider the possibly more challenging task of constructing the free Baxter algebra, not necessarily commutative. In recent interesting work, Ebrahimi-Fard and Guo have successfully tackled this problem [6]; they have in fact constructed the adjoint functor to the forgetful functor from the category of (associative) Baxter algebras to the category of (associative) algebras. As it turns out, there is not much loss of generality in concentrating in the case of one generator $x$, which we do from now on. The construction in [6] involves a certain class of words on the symbols $x$ and $\beta(x)$. This choice of combinatorial structure makes the description of the algebraic structure rather involved and lengthy.

In this paper we provide a simpler description of this algebra, by making use of a different combinatorial structure (decorated trees) and of an appropriate notion of grafting for these objects. We have learned that the authors of [6] were aware of this possibility, and plan to present their results in [7]. Another paper in preparation with related results to ours is [9].

The use of decorated trees makes our construction very reminiscent of the constructions of the free dendriform dialgebra of Loday [10] and of the free dendriform trialgebra of Loday and Ronco [11]. In addition, it allows us to present a unified construction of the free Baxter algebra and of three closely related algebras; namely, that in which the generator $x$ is assumed to be idempotent $\left(x^{2}=x\right)$, that in which the map $\beta$ is assumed to be quasi-idempotent $\left(\beta^{2}=-\lambda \beta\right)$, and that in which both assumptions are made. We refer to any of these as a free Baxter algebra (of the appropriate kind) and denote them by $B_{i, j}^{\lambda}$, where the subindices $i, j \in\{2, \infty\}$ distinguish between the various cases. They are related by a commutative diagram of surjective morphisms of Baxter algebras as follows:



The free Baxter algebras $B_{2, \infty}^{\lambda}$ (in which the generator is assumed to be idempotent) and $B_{\infty, \infty}^{\lambda}$ (in which no assumptions are made) are covered by the adjoint construction 
of [6]. The algebras $B_{2,2}^{\lambda}$ and $B_{\infty, 2}^{\lambda}$ (in which the map is assumed to be quasi-idempotent) constitute the main focus of our work. For our purposes these cases appear to be more fundamental, as explained in the next three paragraphs.

One of our goals is to calculate the dimensions of the homogeneous components of the algebras $B_{i, j}^{\lambda}$, and the corresponding generating series. An important feature is that the combinatorics is captured by the idempotent case: the generating series for the algebras $B_{i, j}^{\lambda}$ are binomial transforms of the generating series for the algebra $B_{2,2}^{\lambda}$. We provide explicit formulas for the dimensions of the homogeneous components of the algebras $B_{2,2}^{\lambda}$ and $B_{\infty, 2}^{\lambda}$, and on the way to these results we provide several bijections between the classes of decorated trees that form linear bases of these algebras and more familiar combinatorial objects, such as planar rooted trees, Schröder paths, and Motzkin paths. For a summary of the most important combinatorial results, see Table 4 .

Another goal is to clarify the connections between free Baxter algebras and free dendriform dialgebras and trialgebras. Dendriform dialgebras and trialgebras were introduced by Loday [10] and Loday and Ronco [11]. A connection between these objects and Baxter algebras was observed in $[1,5]$ : any Baxter algebra with $\lambda=1$ can be turned into a dendriform trialgebra and any Baxter algebra with $\lambda=0$ can be turned into a dendriform dialgebra. This gives rise to morphisms of Baxter algebras from the free dendriform trialgebra on one generator to $B_{\infty, \infty}^{1}$ and from the free dendriform dialgebra on one generator to $B_{\infty, \infty}^{0}$. Ebrahimi-Fard and Guo showed that these maps are injective [6]. We show here that in fact the free dendriform trialgebra embeds in $B_{\infty, 2}^{1}$ and the free dendriform dialgebra embeds in $B_{2,2}^{0}$.

We also discuss algebras $A$ equipped with an idempotent endomorphism of algebras $\beta$. Such a pair $(A, \beta)$ is a Baxter algebra with $\lambda=-1$, so choosing an element of $A$ determines a morphism $B_{\infty, 2}^{-1} \rightarrow A$ of Baxter algebras. We construct the free object on one generator in this category and describe the canonical morphism from $B_{\infty, 2}^{-1}$ in explicit terms. We also provide the analogous results for the case of idempotent generators.

Decorated trees are introduced in Section 2.1, and the notion of grafting, which is central for the construction of the free Baxter algebras, is discussed in 2.2. The construction is carried out in Section 2.3, where we provide a complete concise proof of the universal property of the algebras $B_{i, j}^{\lambda}$ (Proposition 2.4). Section 3 contains the combinatorial results; though our motivation is algebraic, these results are interesting on their own, and they can be read separately from the rest. Section 3.1 presents various kinds of combinatorial objects and then puts them in bijection with the linear bases of the free Baxter algebras. These results are used to calculate the dimensions of the homogeneous components of the free Baxter algebras in Section 3.2, as well as the generating series in 3.3. Algebras with an idempotent endomorphism and their connection to Baxter algebras are discussed in Section 4. The connection with dendriform trialgebras and dialgebras and the embedding results are given in Section 5. The appendix contains two algorithms used to set up some of the bijections of Section 3. 


\section{Notation}

We work over a commutative ring $\mathbb{k}$. By vector space we mean free $\mathbb{k}$-module. All spaces and algebras are over $\mathbb{k}$. All algebras are associative, but not necessarily unital.

The set $\mathbb{Z}^{+}$is the set of positive integers and $\mathbb{N}=\mathbb{Z}^{+} \cup\{0\}$.

\section{Free Baxter algebras on one generator}

Let $A$ be an algebra, $\lambda \in \mathbb{k}$, and $\beta: A \rightarrow A$ a linear map satisfying

$$
\beta(a) \beta(b)=\beta(\beta(a) b+a \beta(b)+\lambda a b)
$$

for all $a, b \in A$. The map $\beta$ is called a Baxter operator and the pair $(A, \beta)$ is called a Baxter algebra of weight $\lambda$. In this case, defining

$$
a *_{\lambda} b=\beta(a) b+a \beta(b)+\lambda a b
$$

one obtains a new associative operation on $A$.

The free Baxter algebra was constructed by Ebrahimi-Fard and Guo [6]. Below we provide another description of the free Baxter algebra on one generator, as well as of three related algebras in which either the generator $x$ is assumed to be idempotent:

$$
x^{2}=x,
$$

or the Baxter map $\beta$ is assumed to be quasi-idempotent:

$$
\beta^{2}=-\lambda \beta
$$

Our description is in terms of decorated trees, as discussed in Section 2.1 below. This allows us to provide simpler definitions of the product in these algebras and of the Baxter maps. It also proves useful in calculating the dimensions of the homogeneous components of these algebras, see Section 3.2.

Remark 2.1. One may wonder about imposing the relation

$$
\beta^{2}=\mu \beta
$$

where $\mu \in \mathbb{k}$ is some scalar other than $-\lambda$. In this case, additional relations follow from (1) and the above relation, such as $\beta(a \beta(b))=\beta(\beta(a) b)=0$ and $\beta(a) \beta(b)=\lambda \beta(a b)$. This leads to three different constructions (according to whether $\lambda=0$ or $\mu=0$ ) which we do not treat in this paper. 


\subsection{Decorated trees}

We define the sets that are going to be bases as vector spaces of the free Baxter algebras.

Consider a rooted planar tree $t$. A node of $t$ is a leaf if it has no children, otherwise it is an internal node. An angle of $t$ is the sector between two consecutive children of an internal node. We decorate $t$ by writing positive integers in the angles and non-negative integers on the internal nodes:

rooted planar tree

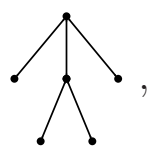

decorated rooted planar tree

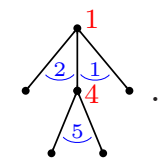

Let $\mathcal{T}_{\infty, \infty}$ be the set consisting of all decorated rooted planar trees satisfying the following conditions:

$\left(R_{1}\right)$ Every internal node has at least two children.

$\left(R_{2}\right)$ Among the children of each node, only the leftmost and rightmost children can be leaves.

$\left(\mathrm{R}_{3}\right)$ Only the root may have label 0; all other internal nodes must be labeled with positive integers.

For example, following trees,
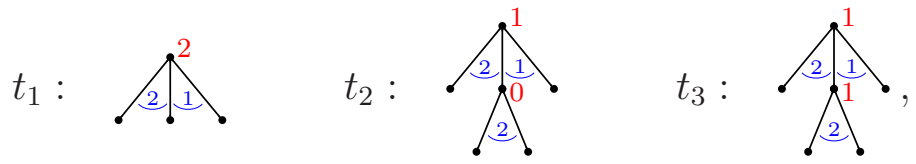

$t_{1}$ verifies conditions $\left(R_{1}\right)$ and $\left(R_{3}\right)$ but does not verify condition $\left(R_{2}\right), t_{2}$ verifies conditions $\left(\mathrm{R}_{1}\right)$ and $\left(\mathrm{R}_{2}\right)$ but not $\left(\mathrm{R}_{3}\right)$, and $t_{3}$ verifies all three conditions.

The subindices in $\mathcal{T}_{\infty, \infty}$ refer to the conditions imposed on the generator and on the Baxter map of the free Baxter algebra, and they will be made clear in Section 2.3.

We define three subsets of $\mathcal{T}_{\infty, \infty}$. Let $\mathcal{T}_{\infty, 2}$ be the subset of $\mathcal{T}_{\infty, \infty}$ consisting of those trees whose internal node labels are less than or equal to 1 . These elements can be seen as trees whose root label is 0 or 1 and the only other decorations are in the angles, since the only possible label for the non-root internal nodes is 1 . Let $\mathcal{T}_{2, \infty}$ be the subset of $\mathcal{T}_{\infty, \infty}$ consisting of those trees whose angle labels are 1 . These elements can be seen as trees whose only decorations are on the internal nodes. Let $\mathcal{T}_{2,2}=\mathcal{T}_{\infty, 2} \cap \mathcal{T}_{2, \infty}$. The set $\mathcal{T}_{2,2}$ consists of two copies of (undecorated) rooted planar trees satisfying conditions $\left(\mathrm{R}_{1}\right)$ and $\left(R_{2}\right)$, where the label 0 or 1 at the root of a tree indicates to which copy it belongs. Table 1 summarizes the decoration rules for each of the four sets.

The following are examples of each kind of tree:

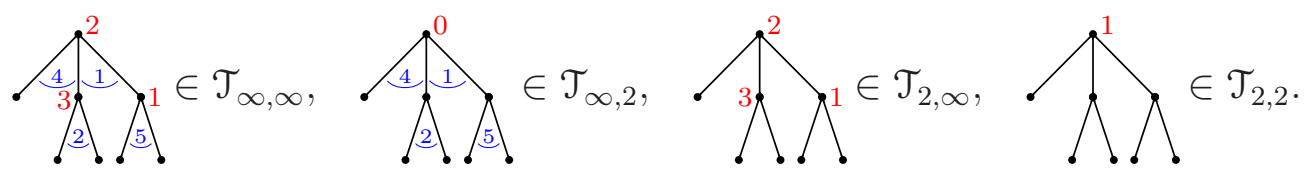




\begin{tabular}{|c||c|c|c|}
\hline Set & Root & Angles & $\begin{array}{c}\text { Non-root } \\
\text { internal nodes }\end{array}$ \\
\hline \hline $\mathcal{T}_{\infty, \infty}$ & $\mathbb{N}$ & $\mathbb{Z}^{+}$ & $\mathbb{Z}^{+}$ \\
$\mathcal{T}_{\infty, 2}$ & $\{0,1\}$ & $\mathbb{Z}^{+}$ & $\{1\}$ \\
$\mathcal{T}_{2, \infty}$ & $\mathbb{N}$ & $\{1\}$ & $\mathbb{Z}^{+}$ \\
$\mathcal{T}_{2,2}$ & $\{0,1\}$ & $\{1\}$ & $\{1\}$ \\
\hline
\end{tabular}

Table 1: Sets of decorated trees

We consider two notions of degree for each kind of tree. The node degree of a decorated tree $t$ is the sum of the labels on the internal nodes of $t$, and we denote it by $\operatorname{deg}_{\text {node }}(t)$. Similarly, the angle degree of $t$, denoted $\operatorname{deg}_{\text {angle }}(t)$, is the sum of the labels in the angles of $t$. Note that $\operatorname{deg}_{\text {angle }}(t)$ is always a positive integer, while degnode may take the value 0 , namely, for the trees $\hat{i}^{0}$.

In particular, observe that for a tree $t$ in $\mathcal{T}_{2, j}$, the angle degree coincides with the number of angles of $t$, which is one less than the number of leaves of $t$. On the other hand, for $t \in \mathcal{T}_{i, 2}$, if the root of $t$ is labeled by 1 then the node degree coincides with the number of internal nodes, while if it is labeled by 0 , the node degree is the number of non-root internal nodes.

For $i, j \in\{2, \infty\}, n \geq 1$, and $m \geq 0$, let

$$
\mathcal{T}_{i, j}(n, m)=\left\{t \in \mathcal{T}_{i, j} \mid \operatorname{deg}_{\text {angle }}(t)=n \text { and } \operatorname{deg}_{\text {node }}(t)=m\right\} .
$$

These sets will be linear bases for the homogeneous components of the free Baxter algebras, see Section 3.2. In Table 2 we show the elements of $\mathcal{T}_{2,2}(n, m)$ for $n=1,2,3$ and $m=$ $0,1,2,3$. We set

$$
\mathcal{T}_{i, j}(*, m)=\bigsqcup_{n \geq 1} \mathcal{T}_{i, j}(n, m), \quad \mathcal{T}_{i, j}(n, *)=\bigsqcup_{m \geq 0} \mathcal{T}_{i, j}(n, m), \quad \mathcal{T}_{i, j}(k)=\bigsqcup_{\substack{n \geq 1, m \geq 0 \\ n+m=k}} \mathcal{T}_{i, j}(n, m)
$$

We let $\mathcal{T}_{i, j}^{+}$(respectively, $\mathcal{T}_{i, j}^{0}$ ) denote the subset of $\mathcal{T}_{i, j}$ consisting of those trees whose root label is positive (respectively, 0), and define $\mathcal{T}_{i, j}^{a}(n, m)=\mathcal{T}_{i, j}^{a} \cap \mathcal{T}_{i, j}(n, m)$, for $a \in$ $\{0,+\}$.

Let $\widehat{\mathcal{T}}_{i, j}=\mathcal{T}_{i, j} \cup\{\cdot\}$ be the set of decorated trees with the (unlabeled) tree with a single node adjoined. We set $\operatorname{deg}(\bullet)=(0,0)$. Similarly, let $\widehat{\mathcal{T}}_{i, j}^{a}=\mathcal{T}_{i, j}^{a} \cup\{\bullet\}$ for $a \in\{0,+\}$.

\subsection{Grafting of decorated trees}

We introduce a grafting operation on the set of decorated trees. Define a function

$$
\mathrm{G}_{i, j}: \bigcup_{n \geq 1}\left(\widehat{\mathcal{T}}_{i, j}^{+}\right)^{n} \times\left(\mathbb{Z}^{+}\right)^{n-1} \longrightarrow \widehat{\mathcal{T}}_{i, j}
$$




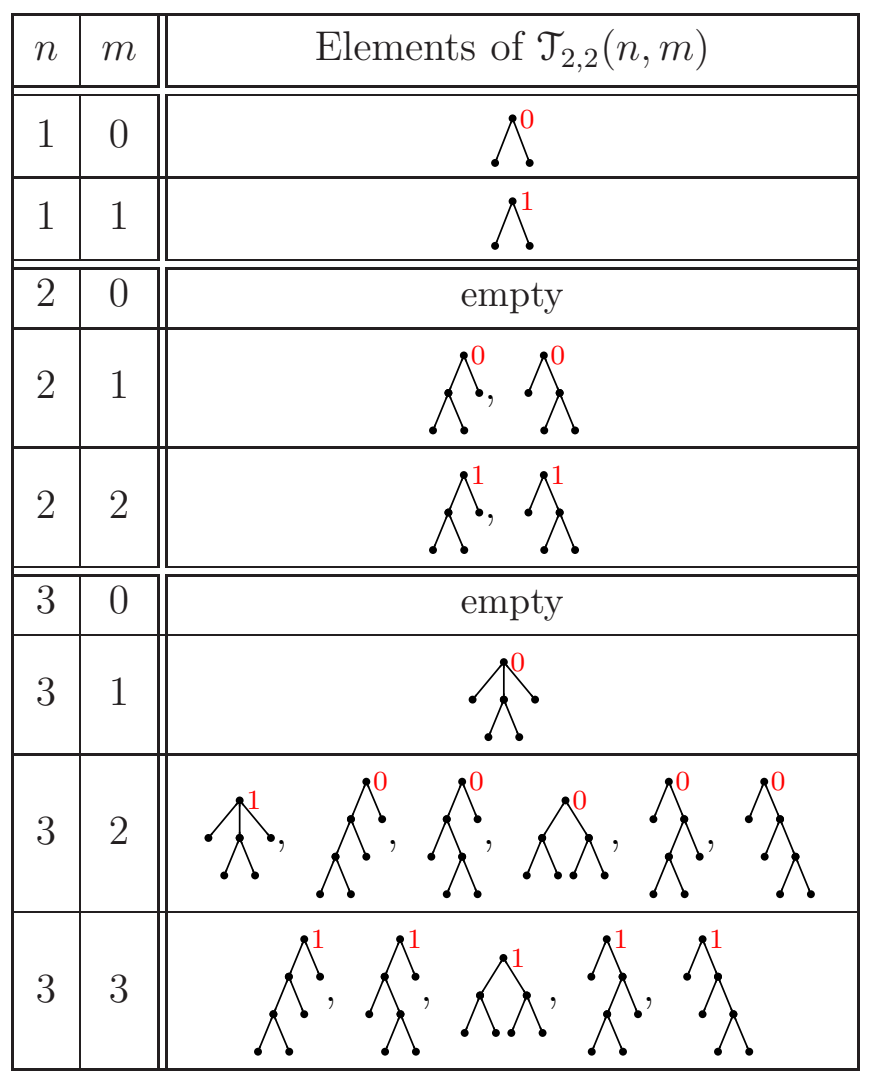

Table 2: $\mathcal{T}_{2,2}(n, m)$ for $n=1,2,3$, and $m=0,1,2,3$

as follows. First, identify the set $\widehat{\mathcal{T}}_{i, j}^{+} \times\left(\mathbb{Z}^{+}\right)^{0}$ with $\widehat{\mathcal{T}}_{i, j}^{+}$and set

$$
\mathrm{G}_{i, j}(t)=t
$$

Then, for $n \geq 2$, set

$$
\mathrm{G}_{i, j}\left(t_{1}, \ldots, t_{n} ; i_{1}, \ldots, i_{n-1}\right)=N(\underbrace{}_{t_{1}}
$$

Here, the function $N$ normalizes the tree in such a way that the result satisfies condition $\left(\mathrm{R}_{2}\right)$; namely, if $t_{k}=\bullet$, for $1<k<n$, then $t_{k}$ and the edge joining it to the new root are removed from the tree, and the two adjacent angles (the angle between $t_{k-1}$ and $t_{k}$ and the one between $t_{k}$ and $t_{k+1}$ ) are merged into one angle which acquires the label $i_{k-1}+i_{k}$. Several additions may occur.

Another clarification is needed. When $i=2$, this addition is performed according to the convention

$$
1+1=1,
$$

so all angle labels remain equal to 1 . (Alternatively, if we view trees in $\mathcal{T}_{2, j}$ as having no angle labels, then no additions are necessary.) 
In other words, for $n>1$, the operation $\mathrm{G}_{i, j}$ grafts the trees $t_{k}$ to a new root with label 0, and uses the arguments $i_{k}$ as the labels of the resulting new angles. Some of these are then added if an intermediate leaf is formed. The result then satisfies conditions $\left(R_{1}\right),\left(R_{2}\right)$, and $\left(R_{3}\right)$, so it is a well-defined element of $\mathcal{T}_{i, j}$.

For example,

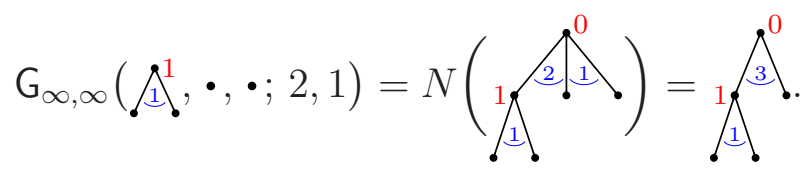

We also define a de-grafting operation $\mathrm{H}_{i, j}: \mathcal{T}_{i, j} \rightarrow \bigcup_{n \geq 1}\left(\widehat{\mathcal{T}}_{i, j}^{+}\right)^{n} \times\left(\mathbb{Z}^{+}\right)^{n-1}$ by

$$
\mathrm{H}_{i, j}(t)= \begin{cases}\left(t_{1}, \ldots, t_{n} ; i_{1}, \ldots, i_{n-1}\right) & \text { if } t \in \mathcal{T}_{i, j}^{0}, \\ t & \text { if } t \in \mathcal{T}_{i, j}^{+}\end{cases}
$$

where for $1 \leq k \leq n, t_{k}$ is the subtree of $t$ rooted at the $k$-th child of the root of $t$ (counting from left to right), and for $1 \leq k \leq n-1, i_{k}$ is the label of the angle between the $k$-th and the $(k+1)$-th children.

For example,

$$
\mathrm{H}_{\infty, \infty}\left(\hat{3}_{1}^{0}\right)=\left(\hat{1}^{1}, \cdot ; 3\right) \text {, while } \mathrm{H}_{\infty, \infty}(\overbrace{1}^{3})=
$$

\subsection{Construction of the free Baxter algebras on one generator}

Let $\mathscr{B}_{\infty, \infty}^{\lambda}$ be the category whose objects are triples $(A, x, \beta)$ where $(A, \beta)$ is a Baxter algebra and $x \in A$ is an element. A morphism $f$ in $\mathscr{B}_{\infty, \infty}^{\lambda}$ from $(A, x, \beta)$ to $(B, y, \gamma)$ is a morphism of algebras that preserves the distinguished elements and commutes with the Baxter operators, that is,

$$
f(x)=y, \quad f \beta=\gamma f .
$$

For $i, j \in\{2, \infty\}$, define $\mathscr{B}_{i, j}^{\lambda}$ as the full subcategory of $\mathscr{B}_{\infty, \infty}^{\lambda}$ whose objects $(A, x, \beta)$ satisfy that

$$
x^{2}=x \text { if } i=2 \text {, and } \beta^{2}=-\lambda \beta \text { if } j=2 .
$$

By the free Baxter algebra on one generator we mean the initial object in the category $\mathscr{B}_{\infty, \infty}^{\lambda}$. The initial object in $\mathscr{B}_{2, \infty}^{\lambda}$ is the free Baxter algebra on one idempotent generator, the initial object in $\mathscr{B}_{\infty, 2}^{\lambda}$ is the free Baxter algebra on one generator and with a quasiidempotent Baxter map, and that in $\mathscr{B}_{2,2}^{\lambda}$ is the free Baxter algebra on one idempotent generator and with a quasi-idempotent Baxter map.

The free Baxter algebra (the initial object in the category $\mathscr{B}_{\infty, \infty}^{\lambda}$ ) was constructed by Ebrahimi-Fard and Guo [6]. The free Baxter algebra on one idempotent generator is also a special case of the constructions of [6]. Below we provide a simpler description of these algebras, as well as of the related algebras mentioned in the preceding paragraph, in a unified manner. 
Definition 2.2. Fix $\lambda \in \mathbb{k}$. Let $B_{i, j}$ the vector space with basis $\mathcal{T}_{i, j}$ and $\widehat{B}_{i, j}$ the vector space with basis $\widehat{\mathcal{T}}_{i, j}$. We extend the map $\mathrm{G}_{i, j}$ of Section 2.2 linearly to these spaces. We define the map $\beta_{i, j}: B_{i, j} \rightarrow B_{i, j}$ as the linear extension of

$$
\beta_{i, j}\left(\Delta^{a}\right)= \begin{cases}\Delta^{a+1}, & \text { when } j \neq 2 \\ (-\lambda)^{a} \Delta^{1}, & \text { when } j=2 .\end{cases}
$$

We also define $\beta_{i, j}(\bullet)=\bullet$ to extend the map to $\beta_{i, j}: \widehat{B}_{i, j} \rightarrow \widehat{B}_{i, j}$.

We define a product $*_{\lambda}$ on the space $\widehat{B}_{i, j}$ and a product $\diamond_{\lambda}$ on the space $B_{i, j}$ by means of a mixed recursion. The recursion starts with

$$
\cdot *_{\lambda} u=u *_{\lambda} \cdot=u
$$

for $u \in \widehat{\mathcal{T}}_{i, j}$, and follows with

$$
t \diamond_{\lambda} s=\mathrm{G}_{i, j}\left(t_{1}, \ldots, t_{n-1}, \beta_{i, j}\left(\bar{t}_{n} *_{\lambda} \bar{s}_{1}\right), s_{2}, \ldots, s_{m} ; i_{1}, \ldots, i_{n-1}, j_{1}, \ldots, j_{m-1}\right),
$$

for $t$ and $s$ in $\mathcal{T}_{i, j}$, and

$$
u *_{\lambda} v=\beta_{i, j}(u) \diamond_{\lambda} v+u \diamond_{\lambda} \beta_{i, j}(v)+\lambda u \diamond_{\lambda} v
$$

for $u, v \in \widehat{\mathcal{T}}_{i, j}$. Here, we have set

$$
\mathbf{H}(t)=\left(t_{1}, \ldots, t_{n} ; i_{1}, \ldots, i_{n-1}\right) \text { and } \mathbf{H}(s)=\left(s_{1}, \ldots, s_{m} ; j_{1}, \ldots, j_{m-1}\right),
$$

and $\bar{t}_{n}$ and $\bar{s}_{1}$ are the result of the operation

$$
\overline{\Delta^{a}}= \begin{cases}\Delta^{a-1}, & \text { if } a>0 ; \\ \bullet, & \text { if } t=\cdot .\end{cases}
$$

Note that $\bar{t}$ is undefined if the root label of $t$ is 0 . In (10), both $t_{n}$ and $s_{1}$ belong to $\widehat{\mathcal{T}}_{i, j}^{+}$, so $\bar{t}_{n}$ and $\bar{s}_{1}$ are well defined. In addition, $\bar{t}_{n} *_{\lambda} \bar{s}_{1}$ involves the computation of products of the form $t^{\prime} \diamond_{\lambda} s^{\prime}$ satisfying $\operatorname{deg}_{\text {node }}\left(t^{\prime}\right) \leq \operatorname{deg}_{\text {node }}(t)$ and $\operatorname{deg}_{\text {node }}\left(s^{\prime}\right) \leq \operatorname{deg}_{\text {node }}(s)$ with at least one of the inequalities being strict. Thus (10) and (11) invoke each other recursively until either $t_{n}=\cdot$ or $s_{1}=\cdot$, at which point the recursion stops with an application of (9). In equation (10) we may encounter a case when $n=1$ (or $m=1$ ). In such a case we understand that the sequence $t_{1}, \ldots, t_{n-1}\left(\right.$ or $\left.s_{2}, \ldots, s_{m}\right)$ is empty, as usual.

By construction, the product $*_{\lambda}$ is related to the product $\diamond_{\lambda}$ and the operator $\beta_{i, j}$ by means of (1). It will then follow, once we show that $\left(B_{i, j}, \diamond_{\lambda}, \beta_{i, j}\right)$ is a Baxter algebra, that $\left(B_{i, j}, *_{\lambda}\right)$ is an associative algebra, with $\left(\widehat{B}_{i, j}, *_{\lambda}\right)$ being its unital augmentation (and with $\bullet$ being the unit element). Note, however, that the product $\diamond_{\lambda}$ is not defined on $\widehat{B}_{i, j}$ and this space is not a Baxter algebra. 
Example 2.3. We illustrate the definition of the product $\diamond_{\lambda}$ with a few small examples. We have $\mathrm{H}\left(\hat{i}^{0}\right)=(\bullet, \bullet ; i)$. Therefore,

$$
\hat{i}^{0} \diamond_{\lambda} \hat{\jmath}^{0}=\mathrm{G}_{\infty, \infty}\left(\cdot, \beta_{\infty, \infty}\left(\cdot *_{\lambda} \cdot\right), \cdot ; i, j\right)=\mathrm{G}_{\infty, \infty}(\cdot, \cdot, \cdot ; i, j)=\widehat{\curlywedge i+j}_{\text {. }}^{0}
$$

Also, since $H\left(\bigwedge_{i}^{1}\right)=\bigwedge_{i}^{1}$, we have

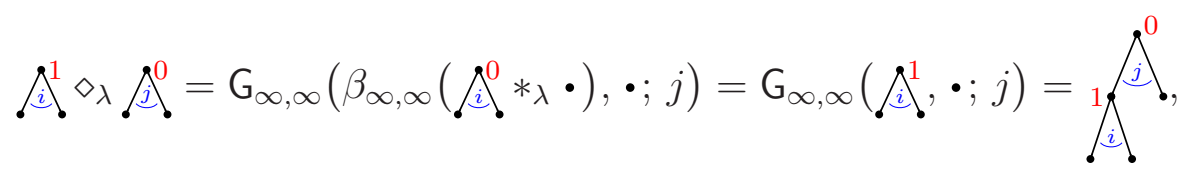

using that $\beta_{\infty, \infty}\left(\bigwedge_{i}^{0}\right)=\AA_{i}^{1}$. With the same considerations we obtain



Finally,



Let $B_{i, j}^{\lambda}$ denote the space $B_{i, j}$ endowed with the product $\diamond_{\lambda}$.

Proposition 2.4. The initial object in the category $\mathscr{B}_{i, j}^{\lambda}$ is $\left(B_{i, j}^{\lambda}, \hat{A}^{0}, \beta_{i, j}\right)$.

Proof. We first consider the case of $\mathscr{B}_{\infty, \infty}^{\lambda}$. This case is dealt with at length in [6], though in a different language. We provide an independent proof to illustrate the efficiency of the notation introduced in this paper. Our arguments extend to cover all categories $\mathscr{B}_{i, j}^{\lambda}$, as discussed at the end of the proof.

During the course of the proof we omit the subindices from the symbols $G_{\infty, \infty}, H_{\infty, \infty}$, $\mathcal{T}_{\infty, \infty}, B_{\infty, \infty}, \widehat{B}_{\infty, \infty}, \mathscr{B}_{\infty, \infty}$, and $\beta_{\infty, \infty}$. Thus, we abbreviate $\mathrm{G}=\mathrm{G}_{\infty, \infty}, \mathrm{H}=\mathrm{H}_{\infty, \infty}$, etc. We also fix de-grafting decompositions of trees $t$ and $s(7)$ as follows:

$$
\mathrm{H}(t)=\left(t_{1}, \ldots, t_{n} ; i_{1}, \ldots, i_{n-1}\right), \quad \mathrm{H}(s)=\left(s_{1}, \ldots, s_{m} ; j_{1}, \ldots, j_{m-1}\right) .
$$

We first check that $\beta$ is a Baxter map. For any $t \in \mathcal{T}$, the root label of $\beta(t)$ is at least 1 , so $\beta(t) \in \mathcal{T}^{+}$and by $(7)$ we have $\mathrm{H}(\beta(t))=\beta(t)$. Using (10) and (11) we obtain

$$
\begin{aligned}
\beta(t) \diamond_{\lambda} \beta(s) & =\mathrm{G}\left(\beta\left(t *_{\lambda} s\right)\right)=\beta\left(t *_{\lambda} s\right) \\
& =\beta\left(\beta(t) \diamond_{\lambda} s+t \diamond_{\lambda} \beta(s)+\lambda t \diamond_{\lambda} s\right),
\end{aligned}
$$

observing that $\overline{\beta(t)}=t$, by definition. Hence, $\beta$ verifies condition (1) and it is a Baxter operator. 
Let $(A, x, \gamma)$ be an object of $\mathscr{B}^{\lambda}$. We formally adjoin two elements $1_{*}$ and 1 to $A$. We declare that $1_{*}$ is a unit element for the product $*$ of $A(1)$, and 1 is a unit element for the given product of $A$, and set

$$
\gamma\left(1_{*}\right)=1
$$

In order to define a map $\varphi: B \rightarrow A$, we first set

$$
\varphi(\cdot)=1_{*},
$$

and then, given $t \in \mathcal{T}$, define $\varphi(t) \in A$ recursively by

$$
\varphi(t)=\gamma\left(\varphi\left(\bar{t}_{1}\right)\right) x^{i_{1}} \gamma\left(\varphi\left(\bar{t}_{2}\right)\right) \cdots x^{i_{n-1}} \gamma\left(\varphi\left(\bar{t}_{n}\right)\right),
$$

where $t_{k}$ and $i_{k}$ are as in (14). When $t \in \mathcal{T}^{+}$, we have $\mathrm{H}(t)=t$, so this definition reads $\varphi(t)=\gamma(\varphi(\bar{t}))$. Applying this to $t=\beta(s)$, where $s \in \mathcal{T}$ is an arbitrary tree, we obtain

$$
\varphi(\beta(s))=\gamma(\varphi(s))
$$

since $\bar{t}=s$ in this case. Thus, $\varphi$ commutes with the Baxter operators.

Next, for $t=\hat{A}^{0}$ we have $\mathrm{H}(t)=(\bullet, \bullet ; 1)$ and hence

$$
\varphi\left(\AA_{1}^{0}\right)=\gamma(\varphi(\bullet)) x^{1} \gamma(\varphi(\cdot))=\gamma\left(1_{*}\right) x \gamma\left(1_{*}\right)=x,
$$

proving that $\varphi$ preserves the distinguished elements of $B$ and $A$.

We now check that $\varphi$ is a morphism of algebras by induction on the bidegrees of $t$ and $s$. We will show that $\varphi$ transforms the products $t \diamond_{\lambda} s$ of $B$ and $t *_{\lambda} s$ of $\widehat{B}$ into the products $\varphi(t) \varphi(s)$ of $A$ and $\varphi(t) * \varphi(s)$ of $\widehat{A}$, where $\widehat{A}$ is $A$ with the unit element $1_{*}$ adjoined. Since $\varphi$ commutes with the Baxter operators, knowing that $\varphi\left(t \diamond_{\lambda} s\right)=\varphi(t) \varphi(s)$ holds up to a certain degree, implies that $\varphi\left(t *_{\lambda} s\right)=\varphi(t) * \varphi(s)$ holds up to the same degree. This will in turn be used to prove the former equality for the next degree.

The base case for the induction occurs when $t=\bullet$ or $s=\bullet$, for which it holds trivially that $\varphi\left(t *_{\lambda} s\right)=\varphi(t) * \varphi(s)$.

Before proceeding with the inductive step, we make a general observation. For a tuple of trees $u=\left(u_{1}, \ldots, u_{n}\right) \in\left(\widehat{\mathcal{T}}^{+}\right)^{n}$ and a tuple of positive integers $\alpha=\left(i_{1}, \ldots, i_{n-1}\right) \in$ $\left(\mathbb{Z}^{+}\right)^{n-1}$, we have

$$
\varphi(\mathrm{G}(u ; \alpha))=\gamma\left(\varphi\left(\bar{u}_{1}\right)\right) x^{i_{1}} \cdots x^{i_{n-1}} \gamma\left(\varphi\left(\bar{u}_{n}\right)\right) .
$$

Indeed, if for all $k=2, \ldots, n-1$ we have $u_{k} \neq \cdot$, then $\mathrm{H}(\mathrm{G}(u ; \alpha))=(u ; \alpha)$ and (18) boils down to (16). On the other hand, assume that $u_{k}=$ - for exactly one $k=2, \ldots, n-1$. Then,

$$
\mathrm{H}(\mathrm{G}(u ; \alpha))=\left(u_{1}, \ldots, u_{k-1}, u_{k+1}, \ldots, u_{n} ; i_{1}, \ldots, i_{k-1}+i_{k}, \ldots, i_{n-1}\right)
$$

and therefore

$$
\varphi(\mathrm{G}(u ; \alpha))=\gamma\left(\varphi\left(\bar{u}_{1}\right)\right) x^{i_{1}} \cdots x^{i_{k-1}+i_{k}} \cdots x^{i_{n-1}} \gamma\left(\varphi\left(\bar{u}_{n}\right)\right),
$$

but as $x^{i_{k-1}+i_{k}}=x^{i_{k-1}} \gamma(\varphi(\bullet)) x^{i_{k}}$, we see that (18) holds for such $u$. A similar argument applies if more than one $u_{k}=\bullet$, and we conclude that (18) holds for any tuple $u \in\left(\widehat{\mathcal{T}}^{+}\right)^{n}$. 
Applying equation (18) to the tuple entering in definition (10), we obtain

$$
\varphi\left(t \diamond_{\lambda} s\right)=\gamma\left(\varphi\left(\bar{t}_{1}\right)\right) x^{i_{1}} \cdots x^{i_{n-1}} \gamma\left(\varphi\left(\bar{t}_{n} *_{\lambda} \bar{s}_{1}\right)\right) x^{j_{1}} \cdots x^{j_{m-1}} \gamma\left(\varphi\left(\bar{s}_{m}\right)\right) .
$$

The bidegrees of $\bar{t}_{n}$ and $\bar{s}_{1}$ are smaller than those of $t$ and $s$, so we can assume inductively that $\varphi\left(\bar{t}_{n} *_{\lambda} \bar{s}_{1}\right)=\varphi\left(\bar{t}_{n}\right) *_{\lambda} \varphi\left(\bar{s}_{1}\right)$. Therefore,

$$
\gamma\left(\varphi\left(\bar{t}_{n} *_{\lambda} \bar{s}_{1}\right)\right)=\gamma\left(\varphi\left(\bar{t}_{n}\right) *_{\lambda} \varphi\left(\bar{s}_{1}\right)\right)=\varphi\left(\bar{t}_{n}\right) \varphi\left(\bar{s}_{1}\right),
$$

the latter equality in view of (1). Substituting in (19) and recalling (16) we obtain $\varphi\left(t \diamond_{\lambda} s\right)=\varphi(t) \varphi(s)$, which completes the induction.

It remains to prove that the product $\diamond_{\lambda}$ is associative. Let $t, s$, and $u \in \mathcal{T}$. In addition to the de-grafting decompositions of $t$ and $s$, let $\mathrm{H}(u)=\left(u_{1}, \ldots, u_{\ell} ; k_{1}, \ldots, k_{\ell-1}\right)$ be that of $u$. Two main cases arise, according to $m$, the number of trees in the decomposition of $s$. If $m=1$, we have

$\mathrm{H}\left(t \diamond_{\lambda} s\right)=\mathrm{HG}\left(t_{1}, \ldots, t_{n-1}, \beta\left(\bar{t}_{n} *_{\lambda} \bar{s}\right) ; i_{1}, \ldots, i_{n-1}\right)=\left(t_{1}, \ldots, t_{n-1}, \beta\left(\bar{t}_{n} *_{\lambda} \bar{s}\right) ; i_{1}, \ldots, i_{n-1}\right)$

and

$\mathrm{H}\left(s \diamond_{\lambda} u\right)=\mathrm{HG}\left(\beta\left(\bar{s} *_{\lambda} \bar{u}_{1}\right), u_{2}, \ldots, u_{\ell} ; k_{1}, \ldots, k_{\ell-1}\right)=\left(\beta\left(\bar{s} *_{\lambda} \bar{u}_{1}\right), u_{2}, \ldots, u_{\ell} ; k_{1}, \ldots, k_{\ell-1}\right)$.

since all intermediate trees in these tuples are different from $\bullet$. Therefore,

$$
\left(t \diamond_{\lambda} s\right) \diamond_{\lambda} u=\mathrm{G}\left(t_{1}, \ldots, \beta\left(\left(\bar{t}_{n} *_{\lambda} \bar{s}\right) *_{\lambda} \bar{u}_{1}\right), \ldots, u_{\ell} ; i_{1}, \ldots, i_{n-1}, k_{1}, \ldots, k_{\ell-1}\right) .
$$

and

$$
t \diamond_{\lambda}\left(s \diamond_{\lambda} u\right)=\mathrm{G}\left(t_{1}, \ldots, \beta\left(\bar{t}_{n} *_{\lambda}\left(\bar{s}_{*_{\lambda}} \bar{u}_{1}\right)\right), \ldots, u_{\ell} ; i_{1}, \ldots, i_{n-1}, k_{1}, \ldots, k_{\ell-1}\right) .
$$

We can assume inductively on the node degree that $\diamond_{\lambda}$ is associative on smaller degree trees, which implies that $*_{\lambda}$ is also associative on those trees. Therefore, $\left(\bar{t}_{n} *_{\lambda} \bar{s}\right) *_{\lambda} \bar{u}_{1}=$ $\bar{t}_{n} *_{\lambda}\left(\bar{s} *_{\lambda} \bar{u}_{1}\right)$ and $\left(t \diamond_{\lambda} s\right) \diamond_{\lambda} u=t \diamond_{\lambda}\left(s \diamond_{\lambda} u\right)$. The base case occurs when some of the trees $t_{n}, s$ or $u_{1}$ equals $\cdot$, and then associativity holds trivially.

If $m>1$, then in the tuples

$$
\left(t_{1}, \ldots, \beta\left(\bar{t}_{n} *_{\lambda} \bar{s}_{1}\right), \ldots, s_{m}\right) \text { and }\left(s_{1}, \ldots, \beta\left(\bar{s}_{m} *_{\lambda} \bar{u}_{1}\right), \ldots, u_{\ell}\right)
$$

the only intermediate trees that may equal - are $\beta\left(\bar{t}_{n} *_{\lambda} \bar{s}_{1}\right)$ and $\beta\left(\bar{s}_{m} *_{\lambda} \bar{u}_{1}\right)$. If neither of them equals $\cdot$ then

$$
\mathbf{H}\left(t \diamond_{\lambda} s\right)=\left(t_{1}, \ldots, \beta\left(\bar{t}_{n} *_{\lambda} \bar{s}_{1}\right), \ldots, s_{m} ; i_{1}, \ldots, i_{n-1}, j_{1}, \ldots, j_{m-1}\right)
$$

and

$$
\mathrm{H}\left(s \diamond_{\lambda} u\right)=\left(s_{1} \ldots, \beta\left(\bar{s}_{m} *_{\lambda} \bar{u}_{1}\right), \ldots, u_{\ell} ; k_{1}, \ldots, k_{\ell-1}\right),
$$

from which it follows that both $\left(t \diamond_{\lambda} s\right) \diamond_{\lambda} u$ and $t \diamond_{\lambda}\left(s \diamond_{\lambda} u\right)$ equal

$$
\mathrm{G}\left(t_{1}, \ldots, \beta\left(\bar{t}_{n} *_{\lambda} \bar{s}_{1}\right), \ldots, \beta\left(\bar{s}_{m} *_{\lambda} \bar{u}_{1}\right), \ldots, u_{\ell} ; i_{1}, \ldots, i_{n-1}, j_{1}, \ldots, j_{m-1}, k_{1}, \ldots, k_{\ell-1}\right) .
$$


It remains to deal with the cases when $\bar{t}_{n} *_{\lambda} \bar{s}_{1}=\bullet$ or $\bar{s}_{m} *_{\lambda} \bar{u}_{1}=\bullet$. We consider the case when $\bar{t}_{n} *_{\lambda} \bar{s}_{1}=\bullet$ and $\bar{s}_{m} *_{\lambda} \bar{u}_{1} \neq \bullet$; the others are similar. We have,

$$
\mathrm{H}\left(t \diamond_{\lambda} s\right)=\left(t_{1}, \ldots, t_{n-1}, s_{2}, \ldots, s_{m} ; i_{1}, \ldots, i_{n-1}+j_{1}, \ldots, j_{m-1}\right)
$$

and

$$
\mathrm{H}\left(s \diamond_{\lambda} u\right)=\left(s_{1}, \ldots, \beta\left(\bar{s}_{m} *_{\lambda} \bar{u}_{1}\right), \ldots u_{\ell} ; j_{1}, \ldots, j_{m-1}, u_{1}, \ldots, u_{\ell-1}\right) .
$$

Hence, both $\left(t \diamond_{\lambda} s\right) \diamond_{\lambda} u$ and $t \diamond_{\lambda}\left(s \diamond_{\lambda} u\right)$ equal

$$
\mathrm{G}\left(t_{1}, \ldots, t_{n-1}, s_{2}, \ldots, \beta\left(\bar{s}_{m} *_{\lambda} \bar{u}_{1}\right), \ldots, u_{\ell} ; i_{1}, \ldots, i_{n-1}+j_{1}, \ldots, j_{m-1}, k_{1}, \ldots, k_{\ell-1}\right) .
$$

This completes the proof of the proposition for the case of $\mathscr{B}_{\infty, \infty}^{\lambda}$.

Most of the preceding proof goes through for the general case of the category $\mathscr{B}_{i, j}^{\lambda}$. To finish the proof, we comment on the few exceptional situations that arise when $i=2$ or $j=2$.

When $i=2$. First note that the element $\hat{1}^{0}$ is indeed idempotent, in view of $(6)$ and (12). Now, in the proof of equation (18) we encounter $i_{k-1}+i_{k}=1+1=1$. However, since $(A, x, \gamma)$ is an object of $\mathscr{B}_{2, j}^{\lambda}$, we have $x^{2}=x$, and therefore $x$ can still be split as $x=x \gamma(\varphi(\bullet)) x$. Thus equation (18) and all the conclusions about the map $\varphi$ are valid.

When $j=2$. First note that $\beta_{i, 2}$ is quasi-idempotent: for any $t \in \mathcal{T}_{i, 2}$, the root label of $\beta_{i, 2}(t)$ is 1 , so by $(8), \beta_{i, 2}\left(\beta_{i, 2}(t)\right)=-\lambda \beta_{i, 2}(t)$. In relation to the case $j=\infty$, only the proofs of (15) and (17) require additional argument. The reason is that, in view of (8), $\beta_{i, 2}(t)$ depends on the root label of $t$, which may be 0 or 1 , and in the latter case we get $\beta_{i, 2}(t)=-\lambda t$. In the former case, $\overline{\beta_{i, 2}(t)}=t$.

The proof of (15) still holds when $t$ and $s$ belong to $\mathcal{T}_{i, 2}^{0}$. Suppose that $t \in \mathcal{T}_{i, 2}^{+}$and $s \in \mathcal{T}_{i, 2}^{0}$ (the other cases are similar). In this case,

$$
\beta_{i, 2}(t) \diamond_{\lambda} \beta_{i, 2}(s)=-\lambda t \diamond_{\lambda} \beta_{i, 2}(s)=-\lambda \mathrm{G}_{i, 2}\left(\beta_{i, 2}\left(\bar{t} *_{\lambda} s\right)\right)=-\lambda \beta_{i, 2}\left(\bar{t} *_{\lambda} s\right) .
$$

On the other hand, $\beta_{i, 2}(t) \diamond_{\lambda} s=-\lambda t \diamond_{\lambda} s$, so

$$
\beta_{i, 2}\left(t *_{\lambda} s\right)=\beta_{i, 2}\left(t \diamond_{\lambda} \beta_{i, 2}(s)\right)=\beta_{i, 2}\left(\mathrm{G}_{i, 2}\left(\beta_{i, 2}\left(\bar{t} *_{\lambda} s\right)\right)\right)=\beta_{i, 2}\left(\beta_{i, 2}\left(\bar{t} *_{\lambda} s\right)\right) .
$$

Since $\beta_{i, 2}$ is quasi-idempotent, we conclude $\beta_{i, 2}(t) \diamond_{\lambda} \beta_{i, 2}(s)=\beta_{i, 2}\left(t *_{\lambda} s\right)$, as needed.

The proof of (17) still holds if $s \in \mathcal{T}_{i, 2}^{0}$. If $s \in \mathcal{T}_{i, 2}^{+}$then we have $\beta_{i, 2}(\bar{s})=s$ and $\beta_{i, 2}(s)=-\lambda s$. Hence,

$$
\varphi \beta_{i, 2}(s)=-\lambda \varphi(s)=-\lambda \varphi \beta_{i, 2}(\bar{s})=-\lambda \gamma \varphi(\bar{s})=\gamma \gamma \varphi(\bar{s})=\gamma \varphi \beta_{i, 2}(\bar{s})=\gamma \varphi(s) .
$$

We used that $\gamma$ is a quasi-idempotent Baxter operator, which holds since in this case $(A, x, \gamma)$ is an object of $\mathscr{B}_{i, 2}^{\lambda}$, and that $\varphi \beta_{i, 2}(\bar{s})=\gamma \varphi(\bar{s})$, which holds since $\bar{s} \in \mathcal{T}_{i, 2}^{0}$. This completes the proof of the proposition. 
Remark 2.5. Consider the forgetful functor from the category of Baxter algebras to the category of algebras. The adjoint functor was constructed by Ebrahimi-Fard and Guo [6]. Applying this functor to the one-dimensional algebra $\mathbb{k}\{x\}\left(x^{2}=x\right)$ yields the algebra $B_{2, \infty}^{\lambda}$, while applying it to the algebra $x \mathbb{k}[x]$ yields $B_{\infty, \infty}^{\lambda}$. Our notation is also useful for describing this functor: simply consider decorated trees in which the angle labels are elements of a given algebra $A$. The notion of grafting naturally extends to this context (using the product of $A$ when a merging of angles occurs in (5)), and the constructions of this section carry through. The result is the value of the adjoint functor on the algebra $A$.

We derive a useful recursive expression for the canonical morphism from the free Baxter algebra to another Baxter algebra.

Corollary 2.6. Let $(A, x, \beta)$ be an object in $\mathscr{B}_{i, j}^{\lambda}$, and $\varphi: B_{i, j}^{\lambda} \rightarrow A$ the unique morphism of Baxter algebras such that $\varphi(\hat{\hat{1}})=x$. Given a tree $t \in \mathcal{T}_{i, j}$, let $a \in \mathbb{N}$ be its root label, $t_{1}, \ldots, t_{n} \in \widehat{\mathcal{T}}_{i, j}^{+}$be the subtrees of $t$ rooted at the children of the root of $t$, and $i_{1}, \ldots, i_{n-1}$ the labels of the angles between these children, as in (7). Then,

$$
\varphi(t)=\gamma^{a}\left(\varphi\left(t_{1}\right) x^{i_{1}} \varphi\left(t_{2}\right) x^{i_{2}} \cdots x^{i_{n-1}} \varphi\left(t_{n}\right)\right) .
$$

In particular, $t$ decomposes as

$$
t=\beta_{i, j}^{a}\left(t_{1} \diamond_{\lambda}\left(\hat{\Lambda}^{0}\right)^{i_{1}} \diamond_{\lambda} t_{2} \diamond_{\lambda}\left(\hat{\Lambda}^{0}\right)^{i_{2}} \diamond_{\lambda} \cdots \diamond_{\lambda}\left(\hat{\Lambda}^{0}\right)^{i_{n-1}} \diamond_{\lambda} t_{n}\right),
$$

where we understand that if $t_{1}$ or $t_{n}$ are equal to $\cdot$ then they are omitted.

Proof. As shown in the proof of Proposition 2.4, the map $\varphi$ is defined by (16). If $a \geq 1$, then $\mathrm{H}_{i, j}(t)=t$, so $\varphi(t)=\gamma(\varphi(\bar{t}))$. The root label of $\bar{t}$ is $a-1$. Proceeding by induction we see that $\varphi(t)=\gamma^{a}(\varphi(\hat{t}))$, where $\hat{t}$ is the same tree as $t$ but with root label 0 . Now, since $\mathrm{H}_{i, j}(\hat{t})=\left(t_{1}, \ldots, t_{n} ; i_{1}, \ldots, i_{n-1}\right)$, and $\gamma\left(\varphi\left(\bar{t}_{k}\right)\right)=\varphi\left(\beta_{i, j}\left(\bar{t}_{k}\right)\right)=\varphi\left(t_{k}\right)$, an application of (16) gives

$$
\varphi(\hat{t})=\gamma\left(\varphi\left(\bar{t}_{1}\right)\right) x^{i_{1}} \gamma\left(\varphi\left(\bar{t}_{2}\right)\right) \cdots x^{i_{n-1}} \gamma\left(\varphi\left(\bar{t}_{n}\right)\right)=\varphi\left(t_{1}\right) x^{i_{1}} \varphi\left(t_{2}\right) x^{i_{2}} \cdots x^{i_{n-1}} \varphi\left(t_{n}\right),
$$

and (20) follows.

Applying this result to $A=B_{i, j}^{\lambda}, x=\hat{\wedge}$, and $\gamma=\beta_{i, j}$ we obtain (20), since in this case $\varphi$ is the identity.

The inclusions among the various categories $\mathscr{B}_{i, j}^{\lambda}$ determine morphisms in the opposite direction among the corresponding initial objects, as indicated below
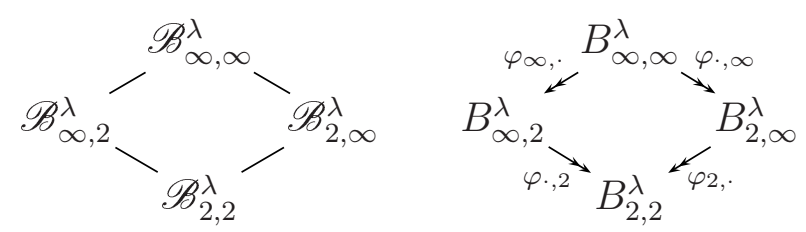

These maps are the unique morphisms of Baxter algebras that preserve the distinguished elements 1. We describe these maps next. 
Proposition 2.7. The maps $\varphi_{\cdot, j}$ are the linearizations of the maps $\mathcal{T}_{\infty, j} \rightarrow \mathcal{T}_{2, j}$ that erase all angle labels. The maps $\varphi_{i,}$, are given by

$$
t \mapsto(-\lambda)^{d} t^{\prime}
$$

where the tree $t^{\prime}$ is obtained from $t$ by changing all positive node labels into 1 , and the exponent $d$ is equal to $\operatorname{deg}_{\text {node }}(t)$ minus the number of nodes of $t$ with positive labels.

Proof. This follows from (8) and (20).

We conclude this section by discussing a canonical filtration on the free Baxter algebras. For each $a \geq 0$, let $B_{i, j}^{\lambda, a}$ be the subspace of $B_{i, j}^{\lambda}$ spanned by those trees $t \in \mathcal{T}_{i, j}$ with root node label less than or equal to $a$. Thus,

$$
B_{i, j}^{\lambda, 0} \subseteq B_{i, j}^{\lambda, 1} \subseteq B_{i, j}^{\lambda, 2} \subseteq \cdots
$$

is an increasing sequence of subspaces of $B_{i, j}^{\lambda}$. Notice also that

$$
\beta_{i, j}\left(B_{i, j}^{\lambda, a}\right) \subseteq B_{i, j}^{\lambda, a+1} .
$$

Define $\widehat{B}_{i, j}^{\lambda, a}$ similarly. According to our definitions, $\bullet \in \widehat{B}_{i, j}^{\lambda, 0}$.

Proposition 2.8. For any $a, b \geq 0$,

$$
\widehat{B}_{i, j}^{\lambda, a} *_{\lambda} \widehat{B}_{i, j}^{\lambda, b} \subseteq \widehat{B}_{i, j}^{\lambda, a+b}, \quad \text { and } B_{i, j}^{\lambda, a} \diamond_{\lambda} B_{i, j}^{\lambda, b} \subseteq \begin{cases}B_{i, j}^{\lambda, a+b-1} & \text { if } a>0 \text { and } b>0, \\ B_{i, j}^{\lambda, 0} & \text { if } a=0 \text { or } b=0 .\end{cases}
$$

In particular, $\widehat{B}_{i, j}^{\lambda, 0}$ is a unital subalgebra of $\left(\widehat{B}_{i, j}^{\lambda}, *_{\lambda}\right)$ and $B_{i, j}^{\lambda, 0}$ is an ideal of $\left(B_{i, j}^{\lambda}, \diamond_{\lambda}\right)$.

Proof. This may be proved by induction, using (9), (10), and (11).

\section{Combinatorics of free Baxter algebras}

\subsection{Trees and paths}

We establish several bijections between the sets of trees defined in Section 2.1 and other combinatorial objects of a more familiar nature. This is used in Section 3.2 to compute the dimensions of the homogeneous components of the free Baxter algebras. The bijections are in the same spirit as those in [17, Proposition 6.2.1].

Let us set the notation for the sets of combinatorial objects. Let $\mathcal{P} \mathcal{T}$ be the set of rooted planar trees whose internal nodes have at least two children. For $n \geq 1$ and $m \geq 0$, let $\mathcal{P} \mathcal{T}(n, m)$ be the subset of $\mathcal{P T}$ consisting of trees with $n+1$ leaves and $m$ internal nodes. Also let $\mathcal{P} \mathcal{T}(n)$ be the set of planar rooted trees with $n+1$ leaves, so that

$$
\mathcal{P T}(n)=\bigsqcup_{m \geq 0} \mathcal{P T}(n, m) .
$$


Observe that $\mathcal{P T}(n, 0)=\emptyset$ for any $n \geq 1$. The cardinality of $\mathcal{P} \mathcal{T}(n)$ is the small Schröder number [17, Exercise 6.39]. For $m \geq 1$, the cardinality of $\mathcal{P} \mathcal{T}(n, m)$ is

$$
\frac{1}{n+1}\left(\begin{array}{c}
n+m \\
m
\end{array}\right)\left(\begin{array}{c}
n-1 \\
m-1
\end{array}\right)
$$

This is also the number of $(n-m)$-dimensional faces of the $(n-1)$-dimensional associahedron [17, Exercise 6.33].

Let $\mathcal{B T}(n)=\mathcal{P T}(n, n)$. This is the subset of $\mathcal{P T}(n)$ consisting of binary trees. Its cardinality is the Catalan number [17, Exercise 6.19]

$$
C(n)=\frac{1}{n+1}\left(\begin{array}{c}
2 n \\
n
\end{array}\right)
$$

This is also the number of vertices of the $(n-1)$-dimensional associahedron.

Next we define various sets of lattice paths.

$\mathcal{C P}(n): \quad$ set of Catalan paths of length $2 n$; that is, lattice paths from $(0,0)$ to $(n, n)$ with steps $H=(1,0)$ and $V=(0,1)$, never rising above the diagonal. The number of these paths is the Catalan number $C(n)$.

$\mathcal{S P}(n)$ : $\quad$ set of Schröder paths of length $2 n$; that is, lattice paths from $(0,0)$ to $(n, n)$ with steps $H=(1,0), V=(0,1)$, and $D=(1,1)$, never rising above the diagonal. The number of these paths is the large Schröder number (twice the small Schröder number) [17, Exercise 6.39].

$\mathcal{S P}(n, m)$ : set of Schröder paths of length $2 n$ with $n-m$ diagonal steps. The number of these paths is given in Proposition 3.8.

$\mathcal{R P}(n, m)$ : set of paths in $\mathcal{S P}(n, m)$ such that each diagonal step is followed by a horizontal step, except if it is the last step. The number of these paths is given in Proposition 3.7.

$\mathcal{M P}(n)$ : set of Motzkin paths of length $n$; that is, lattice paths from $(0,0)$ to $(n, 0)$ with steps $U=(1,1), H=(1,0)$, and $D=(1,-1)$, never crossing below the $x$-axis. The number of these paths is the Motzkin number [17, Exercise 6.38].

$\mathcal{R} \mathcal{M} \mathcal{P}(n)$ : set of paths in $\mathcal{M P}(n)$ such that each horizontal step is followed by an up step, except if it is the last step.

$\mathcal{M P}_{h}(n)$ : set of $h$-colored Motzkin paths from $(0,0)$ to $(n-1,0)$; that is, Motzkin paths whose horizontal steps are colored with one of two colors. The number of these paths is $C(n)$ [16, Exercise (yyy)].

$\mathcal{M} \mathcal{P}_{h u}(n)$ : set of $(h, u)$-colored Motzkin paths from $(0,0)$ to $(n-1,0)$; that is, Motzkin paths whose horizontal and up steps are colored with one of two colors. The number of these paths enters in Proposition 3.7. 
A few examples follow. The letters R and B stand for the colors of the steps.
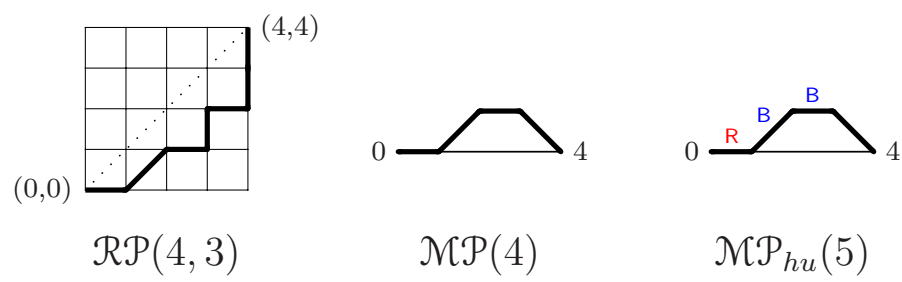

The set of Schröder paths $\mathcal{S P}(n, m)$ and its subset $\mathcal{R P}(n, m)$ can be decomposed into two disjoint subsets:

$$
\mathcal{S P}(n, m)=\mathcal{S P}^{+}(n, m) \sqcup S \mathcal{P}^{0}(n, m), \quad \mathcal{R P}(n, m)=\mathcal{R P}^{+}(n, m) \sqcup \mathcal{R P}^{0}(n, m),
$$

where $\mathcal{S P}^{+}(n, m)$ (respectively $\mathcal{R P}^{+}(n, m)$ ) consists of those paths in $\mathcal{S P}(n, m)$ (respectively $\mathcal{R P}(n, m))$ which do not have diagonal steps lying on the diagonal, and $\mathcal{S P}^{0}(n, m)$ (respectively $\mathcal{R P}^{0}(n, m)$ ) is its complement in $\mathcal{S P}(n, m)$ (respectively $\mathcal{R P}(n, m)$ ).

Proposition 3.1. Let $n \geq 1$ and $m \geq 0$.

(i) The sets $\mathcal{T}_{\infty, 2}^{+}(n, m), \mathcal{P T}(n, m)$, and $\mathcal{S P}^{+}(n, m)$ are in bijection:

$$
\varphi^{+}: \mathcal{T}_{\infty, 2}^{+}(n, m) \stackrel{f^{+}}{\longrightarrow} \mathcal{P T}(n, m) \stackrel{g^{+}}{\longrightarrow} \mathcal{S P}^{+}(n, m) .
$$

(ii) The sets $\mathcal{T}_{\infty, 2}^{0}(n, m), \mathcal{P T}(n, m+1)$, and $\mathcal{S} \mathcal{P}^{0}(n, m)$ are in bijection:

$$
\varphi^{0}: \mathcal{T}_{\infty, 2}^{0}(n, m) \stackrel{f^{0}}{\longrightarrow} \mathcal{P T}(n, m+1) \stackrel{g^{0}}{\longrightarrow} \mathcal{S P}^{0}(n, m) .
$$

Moreover, there is a bijection $T: \mathcal{S P}^{+}(n, m+1) \rightarrow \mathcal{S} \mathcal{P}^{0}(n, m)$ making the following diagram commutative, where $\beta_{\infty, 2}$ is the map that changes the root label from 0 to 1 (Section 2.2),

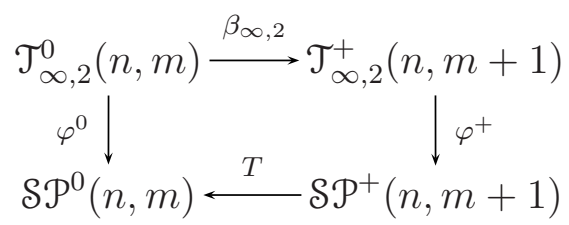

(iii) The bijections $\varphi^{+}$and $\varphi^{0}$ restrict to bijections

$$
\psi^{+}: \mathcal{T}_{2,2}^{+}(n, m) \rightarrow \mathcal{R P}^{+}(n, m), \quad \psi^{0}: \mathcal{T}_{2,2}^{0}(n, m) \rightarrow \mathcal{R P}^{0}(n, m) .
$$

Proof of part (i). Given a tree $t \in \mathcal{T}_{\infty, 2}^{+}(n, m)$, define $f^{+}(t)$ as the planar tree resulting from substituting the decorations $j$ in each angle for $j-1$ intermediate leaves in the corresponding node. The tree $f^{+}(t)$ will have $n$ angles, hence $n+1$ leaves, and $m$ internal nodes, since $\operatorname{deg}_{\text {node }}(t)$, for $t \in \mathcal{T}_{2, \infty}^{+}$, coincides with the number of internal nodes. This process is clearly bijective. For example,

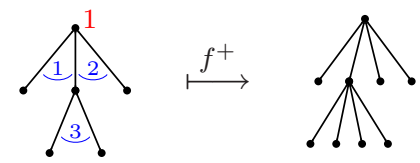


To define the function $g^{+}$, consider a tree $t \in \mathcal{P T}(n, m)$. We generate a Schröder path $p \in \mathcal{S P}^{+}(n, m)$ using Algorithm 1, see Appendix A. Informally, Algorithm 1 traverses the tree depth-first and generates an $H$ step when it finds the leftmost child of a node, a $D$ step when it finds an intermediate child, and a $V$ step when it finds the rightmost child. For example,

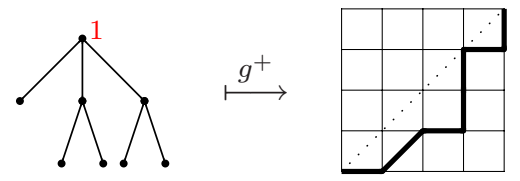

The proof that Algorithm 1 stops is straightforward, since the number of nodes of the trees involved in the recursive invocations of TreeToPath is strictly less than that of $t$.

Note that for each internal node visited by the algorithm, an $H$ step is issued when descending to its leftmost child. Similarly, for each angle a $V$ step or a $D$ step is issued when descending to an intermediate child or to the rightmost child. Therefore, the path contains $m$ horizontal steps, and $n$ steps which are either vertical or diagonal. It can easily be proved by induction on the number of nodes that the algorithm generates an underdiagonal path with the same number of horizontal steps as of vertical steps. Therefore, the path must contain $m$ vertical steps and $n-m$ diagonal steps, and go from $(0,0)$ to $(n, n)$.

We claim that a path generated by Algorithm 1 cannot have a diagonal step lying on the diagonal. Suppose this were the case. The diagonal step cannot be the last step of the path, since Algorithm 1 ends issuing a vertical step. After issuing such a diagonal step, the algorithm processes the tree $t_{i}$. The result is a portion of the path that returns to the diagonal. Eventually a vertical step is issued on exiting the innermost if, which would make the path cross the diagonal. This proves that $g^{+}(t) \in \mathcal{S P}^{+}(n, m)$.

For the reverse process we use Algorithm 2 in Appendix A. From a path in $\mathcal{S P}^{+}(n, m)$ we generate a tree, starting from a single node, by creating the children according to the steps of the path, read from $(0,0)$ to $(n, n)$. If the step is $H$, then a new child is created, the node is marked as available for creating more children, and the algorithm descends to the newly created child. If the step is $D$, then a new child is created in the first available node, searching upward from the current position. The same happens if the step is $V$, but in this case the node where the child is created is marked as no longer available.

It is easy to see, inductively on $n$, that a path in $\mathcal{S P}^{+}(n, m)$ yields a planar tree. Note that the internal nodes are created by horizontal steps, hence there are $m$ such nodes. And the $D$ and $V$ steps produce angles, thus there are $(n-m)+m=n$ angles. Therefore, the output of Algorithm 2 is a tree in $\mathcal{P T}(n, m)$. Clearly, the two algorithms are inverse of each other.

Proof of part (ii). We define the map $T: \mathcal{S P}^{+}(n, m+1) \rightarrow \mathcal{S P}^{0}(n, m)$ shown in Diagram (23) as follows. Let $p=s_{1} s_{2} \cdots s_{k}$ be a path in $\mathcal{S P}^{+}(n, m+1)$. Since a path in $\mathcal{S P}^{+}(n, m+1)$ does not have $D$ steps on the main diagonal, $s_{1}$ and $s_{k}$ must be $H$ and $V$ steps, respectively. Let $p^{\prime}$ be the path obtained from $p$ by dropping the frist and last steps, and shifting the rest by $(-1,0)$. In other words, $p^{\prime}=s_{2} \cdots s_{k-1}$, with origin at 
$(0,0)$ and end at $(n-1, n-1)$. Define

$$
T(p)= \begin{cases}s_{2} \cdots s_{k-1} D & \text { if } p^{\prime} \text { is underdiagonal, } \\ s_{2} \cdots s_{i-1} D H s_{i+1} \cdots s_{j-1} V s_{j+1} \cdots s_{k-1} & \text { if } p^{\prime} \text { crosses the diagonal }\end{cases}
$$

where, in the second case, $s_{i}$ is the first vertical step of $p^{\prime}$ above the diagonal and $s_{j}$ is the last step of $p^{\prime}$ to the left of, or on, the diagonal. In both cases, the path $T(p)$ goes from $(0,0)$ to $(n, n)$, is underdiagonal and has one more diagonal step than $p^{\prime}$. Since the original path $p$ has $n-(m+1)$ diagonal steps, after applying $T$ we are left with a path with $n-(m+1)+1=n-m$ diagonal steps. It is also clear that the path $T(p)$ always has a $D$ step on the main diagonal. Therefore, $T(p) \in \mathcal{S P}^{0}(n, m)$.

To define the inverse of $T$ observe that if $p^{\prime}$ crosses the diagonal then $T(p)$ does not end in a $D$ step. If this were the case, $s_{k-1}=D$ would be a step of $p^{\prime}$ on the diagonal, which contradicts the choice of $j$. Therefore, the inverse of $T$ can be defined by

$$
T^{-1}(p)= \begin{cases}H s_{2} \cdots s_{k-1} V & \text { if } s_{k}=D \\ H s_{1} \cdots s_{i-1} V s_{i+2} \cdots s_{j-1} H s_{j+1} \cdots s_{k} V & \text { if } s_{k} \neq D\end{cases}
$$

where $s_{i}$ is the first diagonal step on the diagonal and $s_{j}$ is the first vertical step after $s_{i}$ which touches the diagonal. This proves that $T$ is bijective.

Observe that the map $\beta_{\infty, 2}: \mathcal{T}_{\infty, 2}^{0}(n, m) \rightarrow \mathcal{T}_{\infty, 2}^{+}(n, m+1)$ is a bijection. The bijection $\varphi^{0}$ is constructed as

$$
\mathcal{T}_{\infty, 2}^{0}(n, m) \stackrel{\beta_{\infty, 2}}{\longrightarrow} \mathcal{T}_{\infty, 2}^{+}(n, m+1) \stackrel{f^{+}}{\longrightarrow} \mathcal{P T}^{+}(n, m+1) \stackrel{g^{+}}{\longrightarrow} \mathcal{S} \mathcal{P}^{+}(n, m+1) \stackrel{T}{\rightarrow} \mathcal{S P}^{0}(n, m),
$$

which fills Diagram (23).

Proof of (iii). The function $f^{+}$restricted to $\mathcal{T}_{2,2}^{+}(n, m)$ just erases the root labels of the trees. Then, given a tree $t \in \mathcal{T}_{2,2}^{+}$, we only need to verify that every $D$ step in $\varphi^{+}(t)$ is followed by an $H$ step. But after Algorithm 1 issues a $D$ step, the tree $t_{i}$ is processed and since it cannot be a leaf by condition $\left(\mathrm{R}_{2}\right)$ the next issued step must be $H$. Conversely, when processing a $D$ step followed by an $H$ step, Algorithm 2 creates a child of the intermediate node we are visiting, and hence no intermediate node is a leaf. This proves that $\varphi^{+}$restricts to a bijection $\psi^{+}: \mathcal{T}_{2,2}^{+}(n, m) \rightarrow \mathcal{R P}^{+}(n, m)$.

Also, the function $T$ preserves the condition that $D$ steps are followed by $H$ steps, then Diagram (23) shows that $\varphi^{0}$ restricts to a bijection $\psi^{0}: \mathcal{T}_{2,2}^{0}(n, m) \rightarrow \mathcal{R P}^{0}(n, m)$.

Remark 3.2. The bijections of Proposition 3.1 give a description for the number of $(n-m)$-dimensional faces of the $(n-1)$-dimensional associahedron $(22)$ in terms of two classes of Schröder paths (the sets $\mathcal{S P}^{+}(n, m)$ and $\left.\mathcal{S P}^{0}(n, m-1)\right)$. A description in terms of a different class of Schröder paths is given in [3, Proposition 2.7].

Corollary 3.3. (i) For $n \geq 1$ and $m \geq 0$, there are bijections

$$
\begin{aligned}
\mathcal{T}_{\infty, 2}(n, m) \leftrightarrow & \mathcal{P} \mathcal{T}(n, m) \sqcup \mathcal{P T}(n, m+1) \leftrightarrow \mathcal{S P}(n, m), \\
& \mathcal{T}_{2,2}(n, m) \leftrightarrow \mathcal{R} \mathcal{P}(n, m) .
\end{aligned}
$$


(ii) For $n \geq 1$, there are bijections

$$
\begin{gathered}
\mathcal{T}_{\infty, 2}^{+}(n, *) \leftrightarrow \mathcal{T}_{\infty, 2}^{0}(n, *) \leftrightarrow \mathcal{P} \mathcal{T}(n) \leftrightarrow \mathcal{S P}^{+}(n) \leftrightarrow \mathcal{S P}^{0}(n), \\
\mathcal{T}_{\infty, 2}(n, *) \leftrightarrow \mathcal{P} \mathcal{T}(n) \times\{0,+\} \leftrightarrow \mathcal{S P}(n)
\end{gathered}
$$

In particular, $\# \mathcal{S P}^{+}(n)=\# \mathcal{S P}^{0}(n)=$ small Schröder number.

(iii) For $n \geq 1$, there are bijections

$$
\begin{gathered}
\mathcal{T}_{2,2}^{+}(n, *) \leftrightarrow \mathcal{T}_{2,2}^{0}(n, *) \leftrightarrow \mathcal{M P}_{h u}(n) \\
\mathcal{T}_{2,2}(n, *) \leftrightarrow \mathcal{M P}_{h u}(n) \times\{0,+\} .
\end{gathered}
$$

Proof. Parts $(i)$ and $(i i)$ are immediate from Proposition 3.1. For part (iii), we construct the bijection $f: \mathcal{T}_{2,2}^{+}(n, *) \rightarrow \mathcal{M} \mathcal{P}_{h u}(n)$ as follows. Given $t \in \mathcal{T}_{2,2}^{+}$, consider the path $\varphi^{+}(t)=s_{1} s_{2} \cdots s_{k} \in \mathcal{R P}^{+}(n, m)$ for some $m$. We know that $s_{1}=H$ and $s_{k}=V$. Consider the path $p=s_{2} \cdots s_{k-1}$ and start reading it from left to right. Using Table 3, the first time that one of the patterns listed in the left column of the table is found, write the corresponding value of the right column, and continue with the rest of the path. Let $p^{\prime}$ be the resulting path. Consider the increment in the distance to the diagonal, from the start to the end point, for each pattern of $p$. When this increment is 0 , so is the increment of distance to the line $y=0$ in the path $p^{\prime}$. Note that line 8 in Table 3 is one of these cases. The condition that $\varphi^{+}(t)$ does not have diagonal steps lying on the diagonal guarantees that the end point of the down step in $p^{\prime}$ is above the line $y=0$, and thus, the search indicated in line 8 is not empty. When the increment in $p$ is $\pm \sqrt{2}$ (diagonally), the increment in $p^{\prime}$ is \pm 1 (vertically), in each case with the same sign. Observe that for each pattern of $p$ of length $2 k$, the corresponding portion of the path $p^{\prime}$ has length $k$. Hence, the path $p^{\prime}$ goes from $(0,0)$ to $(n-1,0)$, as $p$ has length $2 n-2$. Moreover, after removing the first and last steps of $\varphi^{+}(t), p$ rises above the diagonal by at most $\sqrt{2} / 2$ (diagonally). Since this difference is not enough for the path $p^{\prime}$ to cross below the horizontal line $y=0$, by the previous argument, we conclude that $p^{\prime}$ is a Motzkin path in $\mathcal{M} \mathcal{P}_{h u}(n)$.

For the reverse process it is enough to use Table 3 from right to left, taking into account that patterns that are not in the right column (for example a B-up step followed by a R-up step) come from an application of line 8. After adding an $H$ step at the beginning of the resulting Schröder path, and a $V$ step at the end, we guarantee that the result is in $\mathcal{R P}^{+}(n, m)$ for some $m$. From there use the inverse of $\psi^{+}$to get a tree in $\mathcal{T}_{2,2}^{+}(n, *)$.

Remark 3.4. The bijection (24) in Corollary 3.3 is an extension of the bijection $\mathcal{C P}(n) \leftrightarrow$ $\mathcal{M} \mathcal{P}_{h}(n)$ proposed by Stanley as solution to Exercise (yyy) in [16]. More precisely, consider the function $i: \mathcal{M P}_{h}(n) \hookrightarrow \mathcal{M P}_{h u}(n)$ which sends a Motzkin path with only the horizontal steps colored, to the same Motzkin path with all up steps colored red $(\mathrm{R})$. It is easy to see by looking at Table 3 that the corresponding Schröder path for $i(p), p \in \mathcal{M} \mathcal{P}_{h}(n)$, under the bijection ( iii $)$ in Corollary 3.3, is actually a Catalan path. If we embed $\mathcal{B} \mathcal{T}(n)$ in $\mathcal{T}_{2,2}^{+}(n, *)$ as trees with root label 1 , then the bijection $\psi^{+}$from $($iii $)$in Proposition 3.1 
$[\mathrm{b}]$

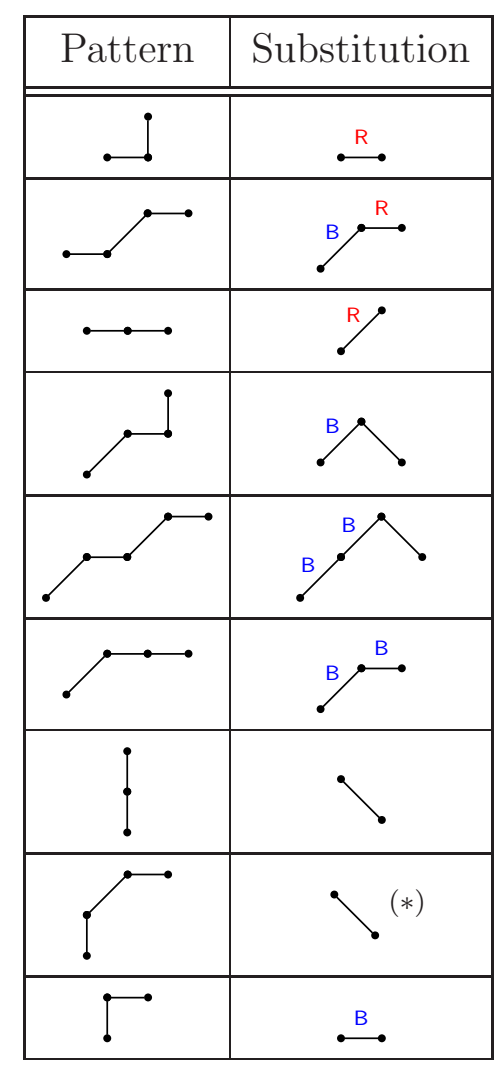

(*) also search backwards the rightmost point where the Motzkin path up-crossed the current level and insert before it the step ${ }^{\mathrm{B}}$.

Table 3: Conversion to colored Motzkin paths.

also restricts and yields the commutative diagram

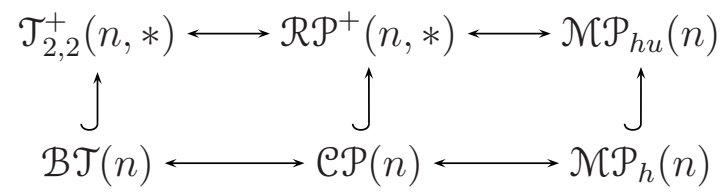

To close this section we study the subspaces $\mathcal{T}_{\infty, 2}(k)$ and $\mathcal{T}_{2,2}(k)$. In this cases, too, it is possible to construct bijections with familiar combinatorial objects, such as Motzkin paths. Recall that

$$
\mathcal{T}_{i, j}(k)=\bigsqcup_{\substack{n \geq 1, m \geq 0 \\ n+m=k}} \mathcal{T}_{i, j}(n, m) .
$$

Proposition 3.5. For $k \geq 1$, there are bijections

$$
\mathcal{T}_{\infty, 2}(k) \leftrightarrow \mathcal{M} \mathcal{P}(k), \quad \mathcal{T}_{2,2}(k) \leftrightarrow \mathcal{R} \mathcal{M} \mathcal{P}(k) .
$$

Proof. From a tree $t \in \mathcal{T}_{\infty, 2}(n, m)$, with $n+m=k$, we get the path $p \in \operatorname{SP}(n, m)$ using part $(i)$ of Corollary 3.3. Reflect the path over the diagonal and rotate it clockwise until 
the diagonal becomes horizontal. If we consider each step to be of length 1 , we obtain a Motzkin path whose length is $2 m+(n-m)=n+m=k$, since $p$ has $m$ horizontal steps, $m$ vertical steps, and $n-m$ diagonal steps. For the converse, given a Motzkin path $p$ of length $k$, let $m$ be the number of up steps, which must coincide with the number of down steps. Let $n=k-m$. Then, the number of horizontal steps is $k-2 m=n-m$. After rotating counter-clockwise and reflecting the path along the diagonal we obtain a Schröder path $q$. This path has $m$ horizontal steps, $m$ vertical steps, and $n-m$ diagonal steps. Hence, we conclude that $q \in \mathcal{S P}(n, m)$ with $n+m=k$. Apply again the bijection of Corollary 3.3 to obtain a tree in $\mathcal{T}_{\infty, 2}(n, m)$.

The second bijection is just the restriction of the previous one. Indeed, the bijection $(i)$ in 3.3 restricts to $\mathcal{T}_{2,2}(n, m) \rightarrow \mathcal{R P}(n, m)$ and the condition about diagonal steps followed by horizontal steps translates, after the geometric transformations, to a condition about horizontal steps followed by up steps.

\subsection{Bigrading and dimensions of the homogeneous components}

Recall the functions degangle $: \mathcal{T}_{i, j} \rightarrow \mathbb{Z}^{+}$and $\operatorname{deg}_{\text {node }}: \mathcal{T}_{i, j} \rightarrow \mathbb{N}$ defined in Section 2.1. Consider the bigrading on the vector space $B_{i, j}$ defined by

$$
\operatorname{deg}(t)=\left(\operatorname{deg}_{\text {angle }}(t), \operatorname{deg}_{\text {node }}(t)\right) .
$$

Let $B_{i, j}(n, m)$ denote the homogeneous component of bidegree $(n, m)$, so that the set $\mathcal{T}_{i, j}(n, m)$ is a basis for $B_{i, j}(n, m)$ and

$$
B_{i, j}=\bigoplus_{\substack{n \geq 1 \\ m \geq 0}} B_{i, j}(n, m)
$$

Similarly, let

$$
B_{i, j}(n, *)=\bigoplus_{m \geq 0} B_{i, j}(n, m), \quad B_{i, j}(*, m)=\bigoplus_{n \geq 1} B_{i, j}(n, m),
$$

and

$$
B_{i, j}(k)=\bigoplus_{\substack{n \geq 1, m \geq 0 \\ n+m=k}} B_{i, j}(n, m) .
$$

Thus, $B_{i, j}(n, *), B_{i, j}(*, m)$, and $B_{i, j}(k)$ are the subspaces of $B_{i, j}$ spanned by $\mathcal{T}_{i, j}(n, *)$, $\mathcal{T}_{i, j}(*, m)$, and $\mathcal{T}_{i, j}(k)$, respectively.

Formula (26) can be understood as follows: $\operatorname{deg}(t)=(n, m)$ if when writing $t \in B_{i, j}$ as an expression in the symbols $x=\wedge^{0}$ and $\beta_{i, j}$ with respect to the product $\diamond_{\lambda}$, the symbol $x$ occurs exactly $n$ times and the symbol $\beta_{i, j}$ occurs at least $m$ times. For this reason, we have

$$
B_{i, j}\left(n_{1}, *\right) \diamond_{\lambda} B_{i, j}\left(n_{2}, *\right) \subseteq B_{i, j}\left(n_{1}+n_{2}, *\right)
$$

but only

$$
B_{i, j}\left(*, m_{1}\right) \diamond_{\lambda} B_{i, j}\left(*, m_{2}\right) \subseteq \bigoplus_{\ell \leq m_{1}+m_{2}} B_{i, j}(*, \ell)
$$


In other words, the decomposition $B_{i, j}=\bigoplus_{n \geq 0} B_{i, j}(n, *)$ is an algebra grading, and the subspaces $B_{i, j}^{(m)}=\bigoplus_{\ell \leq m} B_{i, j}(*, \ell)$ form an algebra filtration. The map $\beta_{i, j}: B_{i, j} \rightarrow B_{i, j}$ preserves the former grading, and increases the latter filtration degree by 1 :

$$
\beta_{i, j}\left(B_{i, j}(n, *)\right) \subseteq B_{i, j}(n, *), \quad \beta_{i, j}\left(B_{i, j}^{(m)}\right) \subseteq B_{i, j}^{(m+1)} .
$$

For each $n \geq 1$ and $m \geq 0$, consider the dimensions

$$
\begin{aligned}
\mathrm{b}_{i, j}(n, m) & =\operatorname{dim}_{\mathbb{K}} B_{i, j}(n, m), & \mathrm{b}_{i, j}(n, *) & =\operatorname{dim}_{\mathbb{R}} B_{i, j}(n, *), \\
\mathrm{b}_{i, j}(*, m) & =\operatorname{dim}_{\mathbb{K}} B_{i, j}(*, m), & \mathrm{b}_{i, j}(k) & =\operatorname{dim}_{\mathbb{K}} B_{i, j}(k) .
\end{aligned}
$$

Our main goal is to compute these dimensions and to describe how they relate to each other as $i$ and $j$ vary over $\{2, \infty\}$.

Consider first the case $m=0$. A tree $t$ with $\operatorname{deg}_{\text {node }}(t)=0$ has only one internal node (the root), and this one is labeled by 0 . For each $n \geq 1$ there is one such tree in $\mathcal{T}_{\infty, j}$, namely $\AA_{n}^{0}$, with bidegree $(n, 0)$. Therefore, $\mathrm{b}_{\infty, \infty}(n, 0)=\mathrm{b}_{\infty, 2}(n, 0)=1$ for all $n \geq 1$. Similarly,

$$
\mathrm{b}_{2, \infty}(n, 0)=\mathrm{b}_{2,2}(n, 0)= \begin{cases}1 & \text { if } n=1 \\ 0 & \text { if } n>1\end{cases}
$$

Unless explicitly stated, from now on we restrict our attention to $n, m \geq 1$.

Given sequences $a(n)$ and $b(n, m)$ defined for $n, m \geq 1$, the binomial transforms of $a(n)$ and $b(n, m)$, respectively, are the sequences defined by

$$
\begin{aligned}
\operatorname{BT}(a)(n) & =\sum_{k=1}^{n}\left(\begin{array}{l}
n-1 \\
k-1
\end{array}\right) a(k), \\
\mathrm{BT}^{2}(b)(n, m) & =\sum_{k=1}^{n} \sum_{\ell=1}^{m}\left(\begin{array}{l}
n-1 \\
k-1
\end{array}\right)\left(\begin{array}{c}
m-1 \\
\ell-1
\end{array}\right) b(k, \ell) .
\end{aligned}
$$

Clearly, the binomial transform $\mathrm{BT}^{2}(b)$ can be computed as a double binomial transform, in any order: if we let $b_{n}^{1}(m)=\mathrm{BT}(b(\cdot, m))(n)$ and $b_{m}^{2}(n)=\mathrm{BT}(b(n, \cdot))(m)$, then

$$
\mathrm{BT}^{2}(b)(n, m)=\mathrm{BT}\left(b_{n}^{1}\right)(m)=\mathrm{BT}\left(b_{m}^{2}\right)(n) .
$$

The following result says that as $i$ and $j$ vary, the dimensions of the homogeneous components $B_{i, j}(n, m)$ can be determined from the dimensions of $B_{2,2}(n, m)$ by applying binomial transforms.

Proposition 3.6. Consider the sequences $\mathbf{b}_{i, j}(n, m)$ for $n, m \geq 1$. We have

$$
\begin{aligned}
\mathrm{b}_{2, \infty}(n, m) & =\mathrm{BT}\left(\mathrm{b}_{2,2}(n, \cdot)\right)(m) \\
\mathrm{b}_{\infty, 2}(n, m) & =\mathrm{BT}\left(\mathrm{b}_{2,2}(\cdot, m)\right)(n) \\
\mathrm{b}_{\infty, \infty}(n, m) & =\mathrm{BT}^{2}\left(\mathrm{~b}_{2,2}\right)(n, m)=\mathrm{BT}\left(\mathrm{b}_{2, \infty}(\cdot, m)\right)(n)=\mathrm{BT}\left(\mathrm{b}_{\infty, 2}(n, \cdot)\right)(m)
\end{aligned}
$$


Proof. Consider the passage from $b_{2,2}$ to $b_{\infty, 2}$. Recall the map $\mathcal{T}_{\infty, 2} \rightarrow \mathcal{T}_{2,2}$ described in Proposition 2.7. Let $t \in \mathcal{T}_{2,2}(k, m)$. This is a tree with $k$ angles and $\operatorname{deg}_{\text {node }}(t)=m$. For each composition of $n$ with $k$ parts there is one tree $\hat{t} \in \mathcal{T}_{\infty, 2}(n, m)$ in the fiber over $t$ of the map (make the parts of the composition be the angle labels of $\hat{t}$ ). Since the number of such compositions is $\left(\begin{array}{l}n-1 \\ k-1\end{array}\right)$, we obtain

$$
\mathrm{b}_{\infty, 2}(n, m)=\sum_{k=1}^{n}\left(\begin{array}{l}
n-1 \\
k-1
\end{array}\right) \mathrm{b}_{2,2}(k, m)=\operatorname{BT}\left(\mathrm{b}_{2,2}(\cdot, m)\right)(n) .
$$

The other cases are similar.

The dimensions $b_{2,2}$ of the homogeneous components of $B_{2,2}$ admit very explicit descriptions, using the bijections from Section 3.1.

Proposition 3.7. For $n \geq 1$ and $m \geq 0$, the dimension of the homogeneous components of $B_{2,2}$ are given by:

(i) $\mathrm{b}_{2,2}(n, m)=\# \mathcal{R P}(n, m)=C(m)\left(\begin{array}{c}m+1 \\ n-m\end{array}\right)$;

(ii) $\mathrm{b}_{2,2}(n, *)=2 \times \# \mathcal{M P P}_{h u}(n)$;

(iii) $\mathrm{b}_{2,2}(*, m)=2^{m+1} C(m)$.

Proof. Using Corollary $3.3(i)$, the equality $b_{2,2}(n, m)=\# \mathcal{R P}(n, m)$ is immediate. To count the number of these paths, remove the $n-m$ diagonal steps from one of those paths, the remaining steps can be assembled into an underdiagonal path from $(0,0)$ to $(m, m)$ with horizontal and vertical steps only. It is well-known that the number of such paths is the Catalan number $C(m)$ [17, Exercise 6.19.h]. To reconstruct the given path from the Catalan path, since a diagonal step can only be followed by a horizontal step, there are $m+1$ possible places to distribute the $n-m$ diagonal steps: exactly before one of the horizontal steps, or in the last position. This is $\left(\begin{array}{c}m+1 \\ n-m\end{array}\right)$ possibilities, and thus the total number of paths is

$$
C(m)\left(\begin{array}{l}
m+1 \\
n-m
\end{array}\right)
$$

as claimed.

Part $($ ii $)$ is a reformulation of Corollary $3.3($ iii $)$. For part (iii) write, using $(i)$,

$$
\mathrm{b}_{2,2}(*, m)=\sum_{n \geq 1} \mathrm{~b}_{2,2}(n, m)=\sum_{n \geq 1} C(m)\left(\begin{array}{c}
m+1 \\
n-m
\end{array}\right)=C(m) 2^{m+1},
$$

by the binomial theorem.

Observe that $\mathrm{b}_{2,2}(n, m)$ is non-zero only in the region $m \leq n \leq 2 m+1$.

The dimensions $\mathrm{b}_{\infty, 2}$ of the homogeneous components of $B_{\infty, 2}$ also admit simple combinatorial descriptions, in addition to the descriptions in terms of decorated trees or in terms of the binomial transform (Proposition 3.6). 
Proposition 3.8. For $1 \leq m \leq n$, the dimension of the homogeneous components of $B_{\infty, 2}$ are given by:

(i) $\mathrm{b}_{\infty, 2}(n, m)=\# \mathcal{S P}(n, m)=C(m)\left(\begin{array}{c}n+m \\ n-m\end{array}\right)$;

(ii) $\mathrm{b}_{\infty, 2}(n, *)=2 \times \# \mathcal{P T}(n)$, which are the large Schröder numbers;

(iii) $\mathrm{b}_{\infty, 2}(*, m)$ is infinite.

Proof. Use Corollary $3.3(i)$ to conclude $\boldsymbol{b}_{\infty, 2}(n, m)=\# \mathcal{S P}(n, m)$. We proceed similarly as before to count this number. Given a path in $\mathcal{S P}(n, m)$, after removing the $n-m$ diagonal steps, we get a Catalan path. To reconstruct the initial path we need to distribute the $n-m$ diagonal steps in $2 m+1$ places: before one of the $m$ horizontal steps, before one of the $m$ vertical steps, or in the last position of the path. Since there can be many consecutive diagonal steps in each place, the total number is

$$
C(m)\left(\begin{array}{c}
(2 m+1)+(n-m)-1 \\
n-m
\end{array}\right)=C(m)\left(\begin{array}{l}
n+m \\
n-m
\end{array}\right) .
$$

Part (ii) is again a direct consequence of Corollary 3.3 (ii). Part (iii) is clear since the decorations in the angles are arbitrary.

Remark 3.9. The three previous propositions show that the small Schröder numbers are the binomial transform of the $(h, u)$-colored Motzkin numbers, a result stated by D. Callan in [15].

Among the sequences $b_{i, j}(k)$, the case of $b_{\infty, 2}$ again proves to be interesting combinatorially.

Proposition 3.10. For $k \geq 1$, the dimensions of the subspaces $B_{2,2}(k)$ and $B_{\infty, 2}(k)$ are

(i) $\mathrm{b}_{2,2}(k)=\# \mathcal{R} \mathcal{M} \mathcal{P}(k)$,

(ii) $\mathrm{b}_{\infty, 2}(k)=\# \mathcal{M} \mathcal{P}(k)$, which are the Motzkin numbers.

Proof. This is a restatement of Proposition 3.5.

The dimensions $\mathbf{b}_{2, \infty}(n, m)$ and $\mathbf{b}_{\infty, \infty}(n, m)$ do not seem to admit any simpler description than as the iterated binomial transforms of $b_{2,2}$. We mention that the sequences $\mathrm{b}_{2, \infty}(*, m)$ and $\mathrm{b}_{2, \infty}(k)$ appear in [15] as A082298 and A025243, respectively, while the dimensions $\mathbf{b}_{2, \infty}(n, *), \mathbf{b}_{\infty, \infty}(n, *)$ and $\mathbf{b}_{\infty, \infty}(*, m)$ are infinite.

Some of the sequences from Propositions 3.7, 3.8, and 3.10 also appear in [15] as $\mathrm{b}_{2,2}(n, m): \mathrm{A} 068763 ; \quad \mathrm{b}_{2,2}(n, *): \quad \mathrm{A} 071356 ; \quad \mathrm{b}_{2,2}(*, m): \quad \mathrm{A} 025225 ; \quad \mathrm{b}_{\infty, 2}(n, *): \mathrm{A} 006318 ;$ $\mathrm{b}_{2,2}(k): \mathrm{A} 007477 ; \mathrm{b}_{2, \infty}(k): \mathrm{A} 025243$.

Table 4 summarizes the results of this section. 


\begin{tabular}{|c||c|c|c|c|}
\hline & $\cdot(n, m)$ & $\cdot(n, *)$ & $\cdot(*, m)$ & $\cdot(k)$ \\
\hline \hline $\begin{array}{c}\mathrm{b}_{2,2} \\
(\lfloor n / 2\rfloor \leq m \leq n)\end{array}$ & $C(m)\left(\begin{array}{l}m+1 \\
n-m\end{array}\right)$ & $\begin{array}{c}\text { twice the number of } \\
(h, u) \text {-colored Motzkin } \\
\text { paths }\end{array}$ & $2^{m+1} C(m)$ & $\# \mathcal{R} \mathcal{N} \mathcal{P}(k)$ \\
\hline $\begin{array}{c}\mathrm{b}_{\infty, 2} \\
(0 \leq m \leq n)\end{array}$ & $C(m)\left(\begin{array}{l}n+m \\
n-m\end{array}\right)$ & $\begin{array}{c}\text { large Schröder } \\
\text { number }\end{array}$ & $\infty$ & $\begin{array}{c}\text { Motzkin } \\
\text { number }\end{array}$ \\
\cline { 2 - 5 }
\end{tabular}

Table 4: Dimensions of the free Baxter algebras with a quasi-idempotent operator

\subsection{Generating series of the free Baxter algebras}

Given sequences $a(n)$ and $b(n, m)$ defined for $n, m \geq 1$, consider their generating functions $A \in \mathbb{k} \llbracket x \rrbracket$ and $B \in \mathbb{k} \llbracket x, y \rrbracket$, defined by

$$
A(x)=\sum_{n \geq 1} a(n) x^{n}, \quad B(x, y)=\sum_{n \geq 1} \sum_{m \geq 1} b(n, m) x^{n} y^{m} .
$$

The binomial transform (27) has a simple expression in terms of generating functions. If $c=\mathrm{BT}(a)$, then the generating functions $C$ and $A$ are related by

$$
C(x)=A\left(\frac{x}{1-x}\right)
$$

Below we find a closed expression for the generating function of the sequence $b_{2,2}(n, m)$, and then we use Proposition 3.6 to obtain the generating functions for the sequences $\mathrm{b}_{2, \infty}(n, m), \mathrm{b}_{\infty, 2}(n, m)$, and $\mathrm{b}_{\infty, \infty}(n, m)$.

Proposition 3.11. The generating functions for the sequences $\mathbf{b}_{i, j}(n, m)$, with $i, j \in$ $\{2, \infty\}$, are as follows:

$$
\begin{aligned}
\mathrm{B}_{2,2}(x, y) & =(1+x) f(x y(1+x)), \\
\mathrm{B}_{2, \infty}(x, y) & =(1+x) f\left(\frac{x y(1+x)}{1-y}\right), \\
\mathrm{B}_{\infty, 2}(x, y) & =\frac{1}{1-x} f\left(\frac{x y}{(1-x)^{2}}\right), \\
\mathrm{B}_{\infty, \infty}(x, y) & =\frac{1}{1-x} f\left(\frac{x y}{(1-x)^{2}(1-y)}\right),
\end{aligned}
$$

where $f(u)=\sum_{n \geq 1} C(n) u^{n}=(1-\sqrt{1-4 u}) /(2 u)-1$ is the generating function for the Catalan numbers.

Proof. The last three formulas follow from the first in view of (28). To verify the first we 
compute

$$
\begin{aligned}
\mathrm{B}_{2,2}(x, y) & =\sum_{m \geq 1} \sum_{n=m}^{2 m+1} C(m)\left(\begin{array}{c}
m+1 \\
n-m
\end{array}\right) x^{n} y^{m} \\
& =\sum_{m \geq 1} C(m)(x y)^{m} \sum_{k=0}^{m+1}\left(\begin{array}{c}
m+1 \\
k
\end{array}\right) x^{k} \\
& =\sum_{m \geq 1} C(m)(x y)^{m}(1+x)^{m+1} \\
& =(1+x) f(x y(1+x)),
\end{aligned}
$$

as claimed.

The generating functions for the sequences $\mathrm{b}_{i, j}(k)$ are obtained by setting $x=y$ in Proposition 3.11.

\section{Free algebra with an idempotent morphism}

Let $A$ be an algebra and $\beta: A \rightarrow A$ an idempotent morphism of algebras:

$$
\beta(a) \beta(b)=\beta(a b), \quad \beta(\beta(a))=\beta(a) .
$$

Then also

$$
\beta(a) \beta(b)=\beta(\beta(a) b+a \beta(b)-a b),
$$

so $\beta$ is an idempotent Baxter operator of weight $\lambda=-1$. We may thus consider the full subcategory $\mathcal{M}_{\infty}$ of $\mathscr{B}_{\infty, 2}^{-1}$ whose objects are triples $(A, x, \beta)$ where $A$ is an algebra, $x \in A$, and $\beta: A \rightarrow A$ is an idempotent morphism of algebras. We refer to the initial object in this category as the free algebra on one generator with an idempotent morphism.

Similarly, by the free algebra with an idempotent morphism and an idempotent generator we mean the initial object in the full subcategory $\mathcal{M}_{2}$ of $\mathcal{M}_{\infty}$ whose objects satisfy $x^{2}=x$.

Since $\mathcal{M}_{\infty}$ is a subcategory of $\mathscr{B}_{\infty, 2}^{-1}$, there is a unique morphism of Baxter algebras from $\left(B_{\infty, 2}^{-1}, \hat{\Lambda}^{0}, \beta_{\infty, 2}\right)$, the initial object in the category $\mathscr{B}_{\infty, 2}^{-1}$, to the initial object in $\mathcal{M}_{\infty}$. Similarly, there is a unique morphism of Baxter algebras from $\left(B_{2,2}^{-1}, \hat{1}_{0}^{0}, \beta_{2,2}\right)$ to the initial object in $\mathcal{M}_{2}$. We proceed to construct these initial objects and to describe these canonical morphisms in explicit terms.

\subsection{Construction of the free algebras with an idempotent mor- phism}

Let $M_{\infty}=\mathbb{k}\left\langle x_{0}, x_{1}\right\rangle$ be the free associative algebra in two variables $x_{0}$ and $x_{1}$. For consistence with the preceding constructions, we stick to the world of non-unital algebras 
(thus, we leave the constant polynomials out). As explained in Remark 4.4 (below), this is not an essential restriction in this context.

Let $\beta_{\infty}: M_{\infty} \rightarrow M_{\infty}$ be the unique morphism of algebras such that

$$
\beta_{\infty}\left(x_{0}\right)=x_{1}, \quad \beta_{\infty}\left(x_{1}\right)=x_{1} .
$$

Proposition 4.1. The initial object in the category $\mathcal{M}_{\infty}$ is $\left(M_{\infty}, x_{0}, \beta_{\infty}\right)$.

Proof. The map $\beta_{\infty}$ is idempotent on the generators, hence everywhere. Therefore, the object $\left(M_{\infty}, x_{0}, \beta_{\infty}\right)$ belongs to the category $\mathcal{M}_{\infty}$.

Let $(A, x, \beta)$ be another object in $\mathcal{M}_{\infty}$. Let $\varphi: M_{\infty} \rightarrow A$ be the unique morphism of algebras such that

$$
\varphi\left(x_{0}\right)=x, \quad \varphi\left(x_{1}\right)=\beta(x) .
$$

We have

$$
\beta \varphi\left(x_{0}\right)=\beta(x)=\varphi\left(x_{1}\right)=\varphi \beta_{\infty}\left(x_{0}\right)
$$

and

$$
\beta \varphi\left(x_{1}\right)=\beta \beta(x)=\beta(x)=\varphi\left(x_{1}\right)=\varphi \beta_{\infty}\left(x_{1}\right),
$$

since $\beta$ is idempotent. Since all these maps are morphisms of algebras, we get that

$$
\beta \varphi=\varphi \beta_{\infty} .
$$

Thus $\left(M_{\infty}, x_{0}, \beta_{\infty}\right)$ is the initial object in $\mathcal{M}_{\infty}$.

In particular, $\left(M_{\infty}, \beta_{\infty}\right)$ is a Baxter algebra. Let $\pi_{\infty}: B_{\infty, 2}^{-1} \rightarrow M_{\infty}$ be the unique morphism of Baxter algebras such that

$$
\pi_{\infty}\left(\left.\hat{1}\right|^{0}\right)=x_{0}
$$

We have

$$
\pi_{\infty}\left(\hat{\mathbf{1}}^{1}\right)=\pi_{\infty}\left(\beta_{\infty, 2}\left(\hat{\mathbf{1}}^{0}\right)\right)=\beta_{\infty}\left(\pi_{\infty}\left(\hat{\Lambda}^{0}\right)\right)=\beta_{\infty}\left(x_{0}\right)=x_{1} .
$$

More generally:

Lemma 4.2. Let $t \in \mathcal{T}_{\infty, 2}, t_{1}, \ldots, t_{n} \in \widehat{\mathcal{T}}_{\infty, 2}^{+}$the subtrees of $t$ rooted at the children of the root, and $i_{1}, \ldots, i_{n-1}$ the labels of the angles between these children, as in (7). Then

$$
\pi_{\infty}(t)= \begin{cases}x_{1}^{\operatorname{deg}_{\text {angle }}\left(t_{1}\right)} x_{0}^{i_{1}} \cdots x_{0}^{i_{n-1}} x_{1}^{\operatorname{deg}_{\text {angle }}\left(t_{n}\right)} & \text { if } t \in \mathcal{T}_{\infty, 2}^{0}, \\ x_{1}^{\operatorname{deg}_{\text {angle }}(t)} & \text { if } t \in \mathcal{T}_{\infty, 2}^{+} .\end{cases}
$$

Proof. We argue by induction on the bidegree of $t$, starting from (29). According to (20), we have

$$
\pi_{\infty}(t)=\beta_{\infty}^{a}\left(\pi_{\infty}\left(t_{1}\right) x_{0}^{i_{1}} \pi_{\infty}\left(t_{2}\right) x_{0}^{i_{2}} \cdots x_{0}^{i_{n-1}} \pi_{\infty}\left(t_{n}\right)\right),
$$

where $a=0$ if $t \in \mathcal{T}_{\infty, 2}^{0}$ and $a=1$ if $t \in \mathcal{T}_{\infty, 2}^{+}$. Now, the trees $t_{k}$ belong to $\mathcal{T}_{\infty, 2}^{+}$and have smaller degree than $t$, so by induction hypothesis $\pi_{\infty}\left(t_{k}\right)=x_{1}^{\operatorname{deg}_{\text {angle }}\left(t_{k}\right)}$ (if $t_{1}=\bullet$ or $t_{n}=\bullet$, then they do not appear in the above expression). Substituting above we get

$$
\pi_{\infty}(t)=\beta_{\infty}^{a}\left(x_{1}^{\operatorname{deg}_{\text {angle }}\left(t_{1}\right)} x_{0}^{i_{1}} x_{2}^{\text {degangle }\left(t_{2}\right)} x_{0}^{i_{2}} \cdots x_{0}^{i_{n-1}} x_{n}^{\text {degangle }\left(t_{n}\right)}\right) .
$$

Using that $\beta_{\infty}$ is a morphism and $\beta_{\infty}\left(x_{0}\right)=x_{1}$ we obtain (31). 
In order to describe the kernel of $\pi_{\infty}$, we introduce the following relation among decorated trees. Recall that the elements of $\mathcal{T}_{\infty, 2}$ are trees whose root label is 0 or 1 and the only other decorations are in the angles. Given $t$ and $s$ in $\mathcal{T}_{\infty, 2}$, write $t \sim s$ if the following conditions hold:

(a) $t$ and $s$ have the same root label.

(b) If the root label is 1 , then

(i) $\operatorname{deg}_{\text {angle }}(t)=\operatorname{deg}_{\text {angle }}(s)$.

If the root label is 0 , then

(i) $t$ and $s$ have the same number of children of the root,

(ii) $\operatorname{deg}_{\text {angle }}\left(t_{k}\right)=\operatorname{deg}_{\text {angle }}\left(s_{k}\right)$ for all $k=1, \ldots, n$,

(iii) $i_{k}=j_{k}$ for all $k=1, \ldots, n-1$,

where $t_{1}, \ldots, t_{n}$ are the subtrees of $t$ rooted at the children of the root, and $i_{1}, \ldots, i_{n-1}$ are the labels of the angles between these children, as in (7), and similarly for $s_{k}, j_{k}$, and $s$.

For example, the following trees are related:

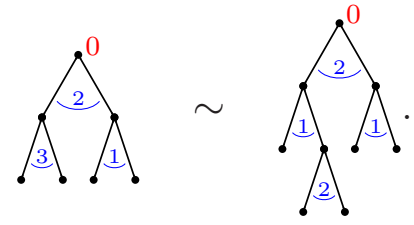

Proposition 4.3. The vector subspace I of $B_{\infty, 2}^{-1}$ spanned by the differences $t-s$ for $t \sim s$ in $\mathcal{T}_{\infty, 2}$ is a Baxter ideal, and

$$
M_{\infty} \cong B_{\infty, 2}^{-1} / I \text {. }
$$

Proof. Consider the morphism $\pi_{\infty}: B_{\infty, 2}^{-1} \rightarrow M_{\infty}$. According to (29) and (30), the algebra generators of $M_{\infty}$ are in its image, so this map is surjective. Now, by Lemma $4.2, \pi_{\infty}$ sends the basis $\mathcal{T}_{\infty, 2}$ of $B_{\infty, 2}^{-1}$ to the basis of words in $x_{0}$ and $x_{1}$ of $M_{\infty}$, from which it follows that the kernel of $\pi_{\infty}$ is the subspace spanned by the differences $t-s$, for all $t, s \in \mathcal{T}_{\infty, 2}$ with $\pi_{\infty}(t)=\pi_{\infty}(s)$. Now, the latter occurs precisely when $t \sim s$, by (31).

The free algebra with an idempotent morphism and an idempotent generator admits a similar description. Let $M_{2}$ be the following quotient of $M_{\infty}$ :

$$
M_{2}=\frac{\mathbb{k}\left\langle x_{0}, x_{1}\right\rangle}{\left\langle x_{0}^{2}=x_{0}, x_{1}^{2}=x_{1}\right\rangle} .
$$

The morphism $\beta_{\infty}: M_{\infty} \rightarrow M_{\infty}$ descends to the quotient, giving rise to another idempotent morphism $\beta_{2}: M_{2} \rightarrow M_{2}$, which turns $\left(M_{2}, \beta_{2}\right)$ into a Baxter algebra of weight $\lambda=-1$. Since $x_{0}$ is now idempotent, $\left(M_{2}, x_{0}, \beta_{2}\right)$ is an object of the category $\mathscr{B}_{2,2}^{-1}$, and 
there is a unique morphism of Baxter algebras $\pi_{2}: B_{2,2}^{-1} \rightarrow M_{2}$ such that $\pi_{2}\left(\bigwedge^{0}\right)=x_{0}$. These fit into a commutative diagram of of morphisms of Baxter algebras:

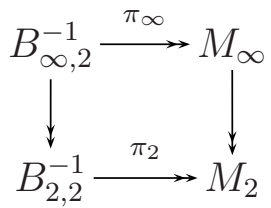

Remark 4.4. For general Baxter algebras, the distinction between the free algebras in the unital case and in the non-unital case is important. In this paper we have dealt with nonunital algebras only. On the other hand, this becomes a minor point when considering the free algebras with an idempotent morphism, as the preceding constructions show. Namely, to obtain the free unital algebras with an idempotent morphism, simply throw in the constant polynomials to the spaces $M_{\infty}$ and $M_{2}$, and extend the morphisms $\beta_{\infty}$ and $\beta_{2}$ so that they preserve the unit element. (Note that for an arbitrary Baxter operator such an extension does not produce another Baxter operator.)

\subsection{Bigrading and dimensions of the free algebras with an idem- potent morphism}

Consider the bigrading of the polynomial algebra $M_{\infty}$ defined as follows. Any monomial $\mu$ in $x_{0}$ and $x_{1}$ can be uniquely written as $\mu=x_{0}^{i_{0}} x_{1}^{j_{1}} x_{0}^{i_{1}} x_{1}^{j_{2}} \ldots x_{1}^{j_{k}} x_{0}^{i_{k}}$ with $i_{0}, i_{k} \geq 0$ and all other exponents $i_{h}, j_{h}>0$. Then set

$$
\operatorname{deg}(\mu)=\left(i_{0}+j_{1}+i_{1}+j_{2}+\cdots+j_{k}+i_{k}, k\right) .
$$

In particular,

$$
\operatorname{deg}\left(x_{0}\right)=(1,0) \text { and } \operatorname{deg}\left(x_{1}\right)=(1,1) .
$$

This can be understood as follows: $\operatorname{deg}(\mu)=(n, m)$ if when writing $\mu \in M_{\infty}$ as a word in $x_{0}$ and $\beta_{\infty}\left(x_{0}\right)$, the symbol $x_{0}$ occurs exactly $n$ times and the symbol $\beta_{\infty}$ occurs at least $m$ times.

Let $M_{\infty}(n, m), M_{\infty}(n, *)$, and $M_{\infty}(*, m)$ be the corresponding homogeneous components. In analogy with the situation encountered for the algebras $B_{i, j}^{\lambda}$ in Section 3.2, we have that the decomposition $M_{\infty}=\bigoplus_{n \geq 0} M_{\infty}(n, *)$ is an algebra grading, but the decomposition $M_{\infty}=\bigoplus_{m \geq 0} M_{\infty}(*, m)$ is not: $x_{1}^{2}$ has degree $(2,1)$. On the other hand, defining $M_{\infty}^{(m)}=\bigoplus_{\ell \leq m} M_{\infty}(*, \ell)$ one obtains an algebra filtration. The map $\beta_{\infty}: M_{\infty} \rightarrow M_{\infty}$ preserves the former grading, and increases the latter filtration degree by $1: \beta_{\infty}\left(M_{\infty}^{(m)}\right) \subseteq$ $M_{\infty}^{(m+1)}$.

Formula (33) also defines a grading on $M_{2}$ (now the exponents $i_{h}$ and $j_{h}$ are at most 1 ), and the quotient map $M_{\infty} \rightarrow M_{2}$ is degree-preserving. In addition, the morphisms $\pi_{\infty}: B_{\infty, 2}^{-1} \rightarrow M_{\infty}$ and $\pi_{2}: B_{2,2}^{-1} \rightarrow M_{2}$ are degree-preserving, in view of (31), so all maps in (32) are degree-preserving morphisms of algebras. We use the same notation for the various homogeneous components of $M_{2}$, and as before, we obtain an algebra grading and 
an algebra filtration on $M_{2}$. The morphism $\beta_{2}$ preserves the former grading and increases the latter filtration degree by 1 .

Let $\mathrm{m}_{\infty}(n, m)=\operatorname{dim}_{\mathbb{R}} M_{\infty}(n, m)$ and $\mathrm{m}_{2}(n, m)=\operatorname{dim}_{\mathbb{R}} M_{2}(n, m)$ be the dimensions of the homogeneous components of bidegree $(n, m)$. It is easy to see that, for any $n, m \geq 0$,

$$
\mathrm{m}_{\infty}(n, m)=\left(\begin{array}{c}
n+1 \\
2 m
\end{array}\right) \text { and } \mathrm{m}_{2}(n, m)= \begin{cases}2 & \text { if } n=2 m \text { and }(n, m) \neq(0,0), \\
1 & \text { if }|n-2 m|=1 \text { or }(n, m)=(0,0), \\
0 & \text { otherwise }\end{cases}
$$

Note that, in analogy with the situation encountered for free Baxter algebras in Proposition 3.6, these dimensions are related by a binomial transform:

$$
\mathrm{m}_{\infty}(n, m)=\operatorname{BT}\left(\mathrm{m}_{2}(\cdot, m)\right)(n)=\sum_{i=1}^{n}\left(\begin{array}{c}
n-1 \\
i-1
\end{array}\right) \mathrm{m}_{2}(i, m) .
$$

This assertion boils down to Pascal's identity for binomial coefficients.

\subsection{Generating series of the free algebras with an idempotent morphism}

We consider the unital version of these algebras. As explained in Remark 4.4, this simply amounts to adding one to the generating series of the non-unital versions. The generating functions for the sequences $\mathrm{m}_{\infty}(n, m)$ and $\mathrm{m}_{2}(n, m), n, m \geq 0$, are easily seen to be

$$
\mathrm{M}_{\infty}(x, y)=\frac{1-x+x y}{(1-x)^{2}-x^{2} y} \text { and } \mathrm{M}_{2}(x, y)=\frac{(1+x)(1+x y)}{1-x^{2} y}
$$

Note that $\mathrm{M}_{\infty}(x, y)=\mathrm{M}_{2}\left(\frac{x}{1-x}, y\right)$, in agreement with (28) and (35).

\section{Connections with dendriform trialgebras and dial- gebras}

\subsection{The free dendriform trialgebra and the free dendriform di- algebra}

Dendriform dialgebras and trialgebras were introduced by Loday [10] and Loday and Ronco [11]. For our purposes it is convenient to consider the following notion.

Definition 5.1. Fix $\lambda \in \mathbb{k}$, a $\lambda$-dendriform trialgebra $D$ is a vector space with three binary operations $\prec, \succ$, and $\cdot$, verifying for all $x, y, z \in D$,

$$
\begin{array}{rlrl}
(x * y) \succ z & =x \succ(y \succ z), & & (x \succ y) \cdot z=x \succ(y \cdot z), \\
(x \succ y) \prec z=x \succ(y \prec z), & & (x \prec y) \cdot z=x \cdot(y \succ z), \\
(x \prec y) \prec z=x \prec(y * z), & & (x \cdot y) \prec z=x \cdot(y \prec z), \\
& (x \cdot y) \cdot z=x \cdot(y \cdot z),
\end{array}
$$


where

$$
x * y=x \prec y+x \succ y+\lambda(x \cdot y) .
$$

For $\lambda=1$ we obtain the usual notion of dendriform trialgebras. For any $\lambda \in \mathbb{k}$, any $\lambda$-dendriform trialgebra may be turned into a 1-dendriform trialgebra by means of the transformation

$$
(D, \prec, \succ, \cdot) \mapsto(D, \prec, \succ, \lambda \cdot)
$$

(multiplying the last operation by $\lambda$ ). If $\lambda \neq 0$, this transformation is invertible, but if $\lambda=0$ a truly distinct notion arises. This notion is closely related to, but not the same as, that of dendriform dialgebras [10]. Specifically, any 0-dendriform trialgebra may be turned into a dendriform dialgebra by means of the transformation

$$
(D, \prec, \succ, \cdot) \mapsto(D, \prec, \succ)
$$

(forgetting the last operation). This transformation is not invertible.

Let $\mathscr{D} \mathscr{T}^{\lambda}$ denote the category whose objects are pairs $(D, x)$ where $D$ is a $\lambda$-dendriform trialgebra and $x \in D$, and whose morphisms are maps that preserve the operations and the distinguished elements.

The initial object in $\mathscr{D} \mathscr{T}^{1}$ (the free dendriform trialgebra on one generator) was constructed in explicit combinatorial terms in [11]. A slight variant of this construction leads to the initial object in $\mathscr{D} \mathscr{T}^{\lambda}$ for any $\lambda \in \mathbb{k}$.

Recall that $\mathcal{P T}$ denotes the set of rooted planar trees, and $\mathcal{P} \mathcal{T}(n, m)$ consists of trees with $n+1$ leaves and $m$ internal nodes (Section 3.1).

Proposition 5.2 ([11]). Let DT be the vector space with basis consisting of the set $\bigoplus_{n, m \geq 1} \mathcal{P T}(n, m)$. Fix $\lambda \in \mathbb{k}$ and define operations on this space by means of the following recursions:

$$
\begin{aligned}
x \prec y & =\mathrm{G}\left(x_{1}, \ldots, x_{n} * y\right), \\
x \succ y & =\mathrm{G}\left(x * y_{1}, y_{2}, \ldots, y_{m}\right), \\
x \cdot y & =\mathrm{G}\left(x_{1}, \ldots, x_{n} * y_{1}, y_{2}, \ldots, y_{m}\right), \\
x * y & =x \prec y+x \succ y+\lambda(x \cdot y) .
\end{aligned}
$$

Let $D T^{\lambda}$ denote the space DT endowed with the operations $\prec, \succ$, and $\cdot$. Then $\left(D T^{\lambda}, \bigwedge\right)$ is the initial object in $\mathscr{D}^{\lambda}$.

In the above definitions, we have set $\mathrm{H}(x)=\left(x_{1}, \ldots, x_{n}\right)$ and $\mathrm{H}(y)=\left(y_{1}, \ldots, y_{m}\right)$, and $\mathrm{G}$ and $\mathrm{H}$ stand for grafting and de-grafting of rooted planar binary trees: $\mathrm{G}$ is defined as in (5) and $\mathrm{H}$ is defined as in the first case of (7), ignoring all labels in both definitions. In contrast to the grafting in (5), no normalization is required, since internal leaves are now allowed. The operation $*$ is defined on the larger space spanned by $\bigoplus_{n, m \geq 0} \mathcal{P T}(n, m)$ and the recursion starts with $x * \bullet=\bullet * x=x$.

Let us consider the analogous notions for dendriform dialgebras. Let $\mathscr{D} \mathscr{D}$ denote the category whose objects are pairs $(D, x)$ where $D$ is a dendriform dialgebra and $x \in D$, and 
whose morphisms are maps that preserve the operations and the distinguised elements. The initial object in $\mathscr{D} \mathscr{D}$ (the free dendriform dialgebra on one generator) was constructed in [10]. On the vector space $D D$ with basis consisting of the set of rooted planar binary trees, two operations $\prec$ and $\succ$ are defined by means of formulas similar to those in Proposition 5.2. The result $(D D, \bigwedge)$ is the initial object in $\mathscr{D} \mathscr{D}$.

\subsection{Embedding dendriform trialgebras and dialgebras in Baxter algebras}

The following observation relates dendriform trialgebras and dialgebras to Baxter algebras.

Proposition $5.3([\mathbf{1}, \mathbf{5}])$. Let $(A, \beta)$ be a Baxter algebra of weight $\lambda$. Defining

$$
x \succ y=\beta(x) y, \quad x \prec y=x \beta(y), \quad \text { and } x \cdot y=x y
$$

one obtains a $\lambda$-dendriform trialgebra structure on $A$.

In view of Proposition 5.3, we may turn the free Baxter algebra $B_{\infty, \infty}^{\lambda}$ into a $\lambda$ dendriform trialgebra. Therefore, there is a unique morphism of dendriform trialgebras

$$
D T^{\lambda} \rightarrow B_{\infty, \infty}^{\lambda}
$$

that sends $\bigwedge$ to $\Lambda_{1}^{0}$. Ebrahimi-Fard and Guo used their construction of the free Baxter algebra to make the interesting observation that this map is injective [6]. Below we derive the stronger fact that the composite

$$
i: D T^{\lambda} \rightarrow B_{\infty, \infty}^{\lambda} \rightarrow B_{\infty, 2}^{\lambda}
$$

is still injective, and describe these map in explicit combinatorial terms.

Proposition 5.4. The canonical morphism of dendriform trialgebras

$$
i: D T^{\lambda} \rightarrow B_{\infty, 2}^{\lambda}
$$

sends any rooted planar tree $x \in \mathcal{P T}$ to the decorated tree $\left(f^{0}\right)^{-1}(x) \in \mathcal{T}_{\infty, 2}^{0}$, where $f^{0}$ is the bijection of Proposition 3.1. In particular, $i$ is injective.

Proof. Let $i^{\prime}: D T^{\lambda} \rightarrow B_{\infty, 2}^{\lambda}$ by the map defined by $i^{\prime}(x)=\left(f^{0}\right)^{-1}(x)$. Then $i^{\prime}(\bigwedge)=\bigwedge_{1}^{0}$. We show below that $i^{\prime}$ is a morphism of dendriform trialgebras; then, by uniqueness, $i^{\prime}=i$.

We proceed by induction on the number of nodes of $x$ and $y$, proving that $i^{\prime}$ preserves the three operations on $D T^{\lambda}$. The equality $i^{\prime}(x \succ x)=i^{\prime}(x) \succ i^{\prime}(x)$, where $x=\bigwedge$ is immediate:

$$
\begin{aligned}
& i^{\prime}(\bigwedge \succ \Lambda)=i^{\prime}(\Lambda)=\hat{\Lambda} \\
& i^{\prime}(\bigwedge) \succ i^{\prime}(\bigwedge)=\beta_{\infty, 2}\left(\Lambda^{0}\right) \diamond_{\lambda} \Lambda_{1}^{0}=\Lambda_{\perp}^{1} \diamond_{\lambda} \hat{\Lambda}^{0}=\hat{\Lambda}^{0} .
\end{aligned}
$$


Similarly we can prove that $i^{\prime}(x \prec x)=i^{\prime}(x) \prec i^{\prime}(x)$ and $i^{\prime}(x * x)=i^{\prime}(x) * i^{\prime}(x)$ in the case $x=\bigwedge$.

The inductive case is similar. Given two trees $x, y$ in $D T^{\lambda}$, we have $i^{\prime}(x \succ y)=$ $i^{\prime}\left(\mathrm{G}\left(x * y_{1}, y_{2}, \ldots, y_{m}\right)\right)$, where $\mathbf{H}(y)=\left(y_{1}, \ldots, y_{m}\right)$. Note that the function $i^{\prime}$ commutes with the grafting operations, in the sense that

$$
i^{\prime}\left(\mathrm{G}\left(t_{1}, \ldots, t_{k}\right)\right)=\mathrm{G}\left(\beta_{\infty, 2} i^{\prime}\left(t_{1}\right), \ldots, \beta_{\infty, 2} i^{\prime}\left(t_{k}\right) ; 1, \ldots, 1\right),
$$

assuming that $i^{\prime}(\cdot)=\boldsymbol{\bullet}$, since the function $\left(f^{0}\right)^{-1}$ and the normalization of the grafting produce the same results when collapsing the intermediate leaves of the trees. Therefore, we can write

$$
\begin{aligned}
i^{\prime}(x \succ y) & =i^{\prime}\left(\mathrm{G}\left(x * y_{1}, \ldots y_{m}\right)\right)=\mathrm{G}\left(\beta_{\infty, 2} i^{\prime}\left(x * y_{1}\right), \ldots, \beta_{\infty, 2} i^{\prime}\left(y_{m}\right) ; 1, \ldots, 1\right) \\
& =\mathrm{G}\left(\beta_{\infty, 2}\left(i^{\prime}(x) * i^{\prime}\left(y_{1}\right)\right), \ldots, \beta_{\infty, 2} i^{\prime}\left(y_{k}\right) ; j_{1}, \ldots, j_{k-1}\right)
\end{aligned}
$$

where in the last equality we have collapsed the intermediate leaves that are children of the root of $y$. Here we used the inductive hypothesis that guarantee $i^{\prime}\left(x * y_{1}\right)=$ $i^{\prime}(x) * i^{\prime}\left(y_{1}\right)$, as $y_{1}$ has less nodes than $y$. On the other hand, to compute $i^{\prime}(x) \succ i^{\prime}(y)=$ $\beta_{\infty, 2}\left(i^{\prime}(x)\right) \diamond_{\lambda} i^{\prime}(y)$, observe that $\overline{\beta_{\infty, 2} i^{\prime}(x)}=i^{\prime}(x)$, since $i^{\prime}(x)$ has root label 0. Also, the de-grafting $\mathrm{H}\left(i^{\prime}(y)\right)$ yields the same subtrees that appear in (40). Thus, using the definition of $\diamond_{\lambda}$ in (10), we conclude that $i^{\prime}(x) \succ i^{\prime}(y)$ coincides with (40). The other operations can be verified similarly.

The map $i^{\prime}$ sends $\Lambda$ to $\hat{A}^{0}$, thus $i^{\prime}=i$ as claimed. The injectivity of $i^{\prime}$ follows easily from the fact that it maps a linear basis of $D T^{\lambda}$ onto a subset of the linear basis $\mathcal{T}_{\infty, 2}^{0}$ of $B_{\infty, 2}^{\lambda}$.

According to Proposition 5.3, a Baxter algebra of weight 0 may be turned into a 0-dendriform trialgebra, which as explained in Section 5.1 gives rise to a dendriform dialgebra. Therefore, there is a unique morphism of dendriform dialgebras

$$
D D \rightarrow B_{\infty, \infty}^{0}
$$

that sends $\bigwedge$ to $\hat{1}$. It is known that this map is injective [6]. In fact, we can show that the composite

$$
j: D D \rightarrow B_{\infty, \infty}^{0} \rightarrow B_{2,2}^{0}
$$

is still injective.

Proposition 5.5. The canonical morphism of dendriform dialgebras

$$
j: D D \rightarrow B_{2,2}^{0},
$$

sends any rooted planar binary tree $x$ to itself with root label 0 . In particular, $j$ is injective. 
Proof. The map $j$ is the composite of the following canonical maps:

$$
D D \hookrightarrow D T^{0} \stackrel{i}{\rightarrow} B_{\infty, 2}^{0} \rightarrow B_{2,2}^{0}
$$

The first map in this chain is the unique morphism of dendriform dialgebras preserving the element $\bigwedge$. It is easy to see from the description of the operations in $D D$ and $D T^{0}$ that this map is simply the linearization of the inclusion of the set of rooted planar binary trees in the set of rooted planar trees. Applied to binary trees, the map $i$ merely adds a label 0 to the root and a label 1 to each angle of the tree, but does not change the underlying tree. The last map in the chain was described in Proposition 2.7; on trees with root label 0 it simply erases the angle labels. Therefore $j$ is as claimed.

Remark 5.6. By Proposition 5.4, the image of the map $i: D T^{\lambda} \hookrightarrow B_{\infty, 2}^{\lambda}$ is precisely the subspace $B_{\infty, 2}^{\lambda, 0}$ of $B_{\infty, 2}^{\lambda}$ spanned by $\mathcal{T}_{\infty, 2}^{0}$. According to Proposition $2.8, B_{\infty, 2}^{\lambda, 0}$ is an ideal for the product $\diamond_{\lambda}$ and a subalgebra for the product $*_{\lambda}$ of $B_{\infty, 2}^{\lambda}$. Moreover, that proposition implies that $B_{\infty, 2}^{\lambda, 0}$ is closed under the dendriform operations of $B_{\infty, 2}^{\lambda}$. Thus, $i$ identifies $D T$ with the dendriform subtrialgebra $B_{\infty, 2}^{\lambda, 0}$ of $B_{\infty, 2}^{\lambda}$. This describes the free dendriform trialgebra explicitly as a subobject of the free Baxter algebra.

The map $j: D D \hookrightarrow B_{2,2}^{0}$ of Proposition 5.5 embeds the free dendriform dialgebra in the dendriform subdialgebra $B_{2,2}^{0,0}$ of $B_{2,2}^{\lambda}$, but its image is strictly smaller.

\subsection{Dendriform dimensions v.s. Baxter dimensions}

Let $D T(n, m)$ be the the subspace of the free $\lambda$-dendriform trialgebra $D T^{\lambda}$ spanned by the set $\mathcal{P T}(n, m)$ (Section 3.1). In other words, a rooted planar tree $x$ has $\operatorname{deg}(x)=(n, m)$ if it has $n+1$ leaves and $m$ internal nodes. In particular, $\operatorname{deg}(\bigwedge)=(1,1)$.

This defines a bigrading on $D T^{\lambda}$ with similar properties to those of the bigrading of the free Baxter algebra $B_{\infty, 2}^{\lambda}$ (Section 3.2). Namely, the dendriform operations preserve the grading defined by the subspaces $D T(n, *)$ and the filtration defined by the subspaces $\bigoplus_{\ell \leq m} D T(*, \ell)$. The morphism $i: D T^{\lambda} \rightarrow B_{\infty, 2}^{\lambda}$ preserves the former grading and decreases the latter filtration degree by 1 , since according to Propositions 3.1 and 5.4, $i$ sends $\mathcal{P} \mathcal{T}(n, m+1)$ to $\mathcal{T}_{\infty, 2}(n, m)$ for $n \geq 1, m \geq 0$.

Consider the dimensions

$$
\begin{gathered}
\operatorname{dt}(n, m)=\operatorname{dim}_{\mathbb{k}} D T(n, m), \quad \operatorname{dt}(n, *)=\operatorname{dim}_{\mathbb{k}} D T(n, *), \\
\text { and } \operatorname{dt}(k)=\operatorname{dim}_{\mathbb{k}} \bigoplus_{\substack{n, m \geq 1 \\
n+m=k}} D T(n, m) .
\end{gathered}
$$

In view of Corollary 3.3, we have the following relation between the dimensions of the homogeneous components of $D T^{\lambda}$ and $B_{\infty, 2}^{\lambda}$ :

$$
\begin{aligned}
\mathrm{b}_{\infty, 2}(n, m)= & \operatorname{dt}(n, m)+\operatorname{dt}(n, m+1), \quad \mathrm{b}_{\infty, 2}(n, *)=2 \mathrm{dt}(n, *) \\
& \text { and } \quad \mathrm{b}_{\infty, 2}(k)=\mathrm{dt}(k)+\mathrm{dt}(k+1) .
\end{aligned}
$$


The first of these relations can be used to deduce the somewhat complicated expression for $\mathrm{dt}(n, m)$ (22) from the simpler expression for $\mathrm{b}_{\infty, 2}(n, m)$ (Proposition 3.8). The second one expresses the relation between the small and the large Schröder numbers, while the last one relates $\mathrm{dt}(k)$ to the Motzkin numbers (Proposition 3.5).

We compare the dimensions of the free dendriform dialgebra $D D$ to those of the free Baxter algebra $B_{2,2}^{0}$. For a planar binary tree $x$ we have a notion of degree, namely, $\operatorname{deg}(x)=n$ if $x$ has $n+1$ leaves. Such a tree has $n$ angles and $n$ internal nodes, so $j(x) \in \mathcal{T}_{2,2}(n, n-1)$ (the root of $j(x)$ has label 0 ). It is well-known that the number of such trees is the Catalan number $C(n)$. Since the map $j$ is injective, it follows from Proposition 3.7 that

$$
C(n) \leq n C(n-1)
$$

for $n \geq 1$. We may view the embedding $j: D D \hookrightarrow B_{2,2}^{0}$ as an algebraic realization of this inequality. 


\section{A Algorithms}

The following algorithms are used in the proof of Proposition 3.1.
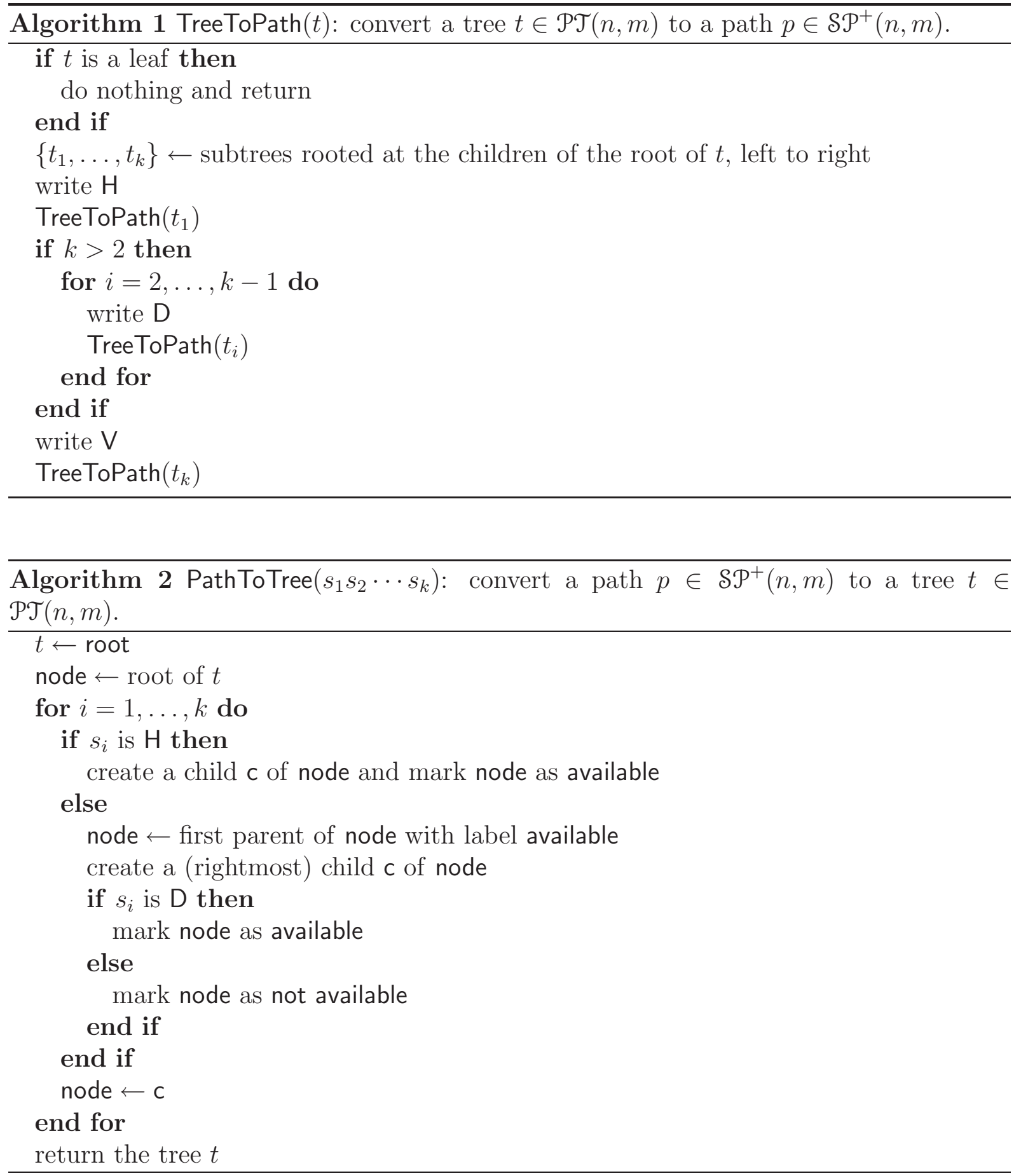


\section{References}

[1] Marcelo Aguiar. Pre-Poisson algebras. Lett. Math. Phys., 54(4):263-277, 2000.

[2] Glen Baxter. An analytic problem whose solution follows from a simple algebraic identity. Pacific J. Math., 10:731-742, 1960.

[3] Joseph Bonin, Louis Shapiro, and Rodica Simion. Some q-analogues of the Schröder numbers arising from combinatorial statistics on lattice paths. J. Statist. Plann. Inference, 34(1):35-55, 1993.

[4] Pierre Cartier. On the structure of free Baxter algebras. Advances in Math., 9:253265, 1972.

[5] Kurusch Ebrahimi-Fard. Loday-type algebras and the Rota-Baxter relation. Lett. Math. Phys., 61(2):139-147, 2002.

[6] Kurusch Ebrahimi-Fard and Li Guo. Rota-Baxter Algebras, Dendriform Algebras and Poincaré-Birkhoff-Witt Theorem. arXiv: math.RA/0503647, 2005.

[7] Li Guo and Kurusch Ebrahimi-Fard. On free Rota-Baxter algebras, 2005.

[8] Li Guo and William Keigher. Baxter algebras and shuffle products. Adv. Math., 150(1):117-149, 2000.

[9] Li Guo and William Sit. Enumeration in free Rota-Baxter algebras, 2005.

[10] Jean-Louis Loday. Dialgebras. In Dialgebras and related operads, volume 1763 of Lecture Notes in Math., pages 7-66. Springer, Berlin, 2001.

[11] Jean-Louis Loday and María Ronco. Trialgebras and families of polytopes. In Homotopy theory: relations with algebraic geometry, group cohomology, and algebraic K-theory, volume 346 of Contemp. Math., pages 369-398. Amer. Math. Soc., Providence, RI, 2004.

[12] Gian-Carlo Rota. Baxter algebras and combinatorial identities. I, II. Bull. Amer. Math. Soc. 75 (1969), 325-329; ibid., 75:330-334, 1969.

[13] Gian-Carlo Rota. Baxter operators, an introduction. In Gian-Carlo Rota on combinatorics, Contemp. Mathematicians, pages 504-512. Birkhäuser Boston, Boston, MA, 1995.

[14] Gian-Carlo Rota. Ten mathematics problems I will never solve. Mitt. Dtsch. Math.Ver., (2):45-52, 1998.

[15] N. J. A. Sloane. The On-Line Encyclopedia of Integer Sequences. published electronically at http://www.research.att.com/ ^njas/sequences/, 2005.

[16] Richard P. Stanley. Catalan addendum. version of 27 May 2005, published electronically at http://www-math.mit.edu/ ${ }^{2}$ stan/ec/catadd.pdf.

[17] Richard P. Stanley. Enumerative combinatorics. Vol. 2, volume 62 of Cambridge Studies in Advanced Mathematics. Cambridge University Press, Cambridge, 1999. With a foreword by Gian-Carlo Rota and appendix 1 by Sergey Fomin. 